\title{
HP1 drives de novo 3D genome reorganization in early Drosophila embryos
}

https://doi.org/10.1038/s41586-021-03460-z

Received: 25 November 2019

Accepted: 16 March 2021

Published online: 14 April 2021

\section{Open access}

Check for updates

\author{
Fides Zenk ${ }^{1,2,7}$, Yinxiu Zhan ${ }^{3,4,7}$, Pavel Kos ${ }^{3}$, Eva Löser ${ }^{1}$, Nazerke Atinbayeva ${ }^{1,2,5}$, \\ Melanie Schächtle', Guido Tiana ${ }^{6}$, Luca Giorgetti ${ }^{3 凶}$ \& Nicola lovino $^{1 \bowtie}$
}

Fundamental features of 3D genome organization are established de novo in the early embryo, including clustering of pericentromeric regions, the folding of chromosome arms and the segregation of chromosomes into active (A-) and inactive (B-) compartments. However, the molecular mechanisms that drive de novo organization remain unknown $\mathrm{n}^{1,2}$. Here, by combining chromosome conformation capture $(\mathrm{Hi}-\mathrm{C})$, chromatin immunoprecipitation with high-throughput sequencing (ChIP-seq), 3D DNA fluorescence in situ hybridization (3D DNA FISH) and polymer simulations, we show that heterochromatin protein 1a (HP1a) is essential for de novo 3D genome organization during Drosophila early development. The binding of HPla at pericentromeric heterochromatin is required to establish clustering of pericentromeric regions. Moreover, HP1a binding within chromosome arms is responsible for overall chromosome folding and has an important role in the formation of B-compartment regions. However, depletion of HP1a does not affect the A-compartment, which suggests that a different molecular mechanism segregates active chromosome regions. Our work identifies HP1a as an epigenetic regulator that is involved in establishing the global structure of the genome in the early embryo.
In metazoans, fertilization triggers global de novo chromatin reorganization into heterochromatin and euchromatin. The clustering of pericentromeric heterochromatin and the folding of chromosome arms lead to a highly regular Rabl configuration during zygotic genome activation (ZGA) $)^{3,4}$. Concomitantly, active and inactive chromatin regions start to associate to form the A- and B-compartments, respectively $^{2,5-9}$. The molecular determinants of compartmental forces remain unknown.

Constitutive heterochromatin is enriched for histone 3 lysine 9 di- and trimethylation ( $\mathrm{H} 3 \mathrm{~K} 9 \mathrm{me} 2 / 3)$ and is important for chromatin structure $^{10,11}$. Members of the heterochromatin protein family bind to constitutive heterochromatin and perform related functions in all eukaryotes $^{12}$. All family members contain a chromodomain ${ }^{13}$, which binds to H3K $9 \mathrm{me} 2 / 3$, and a chromoshadow domain, which supports homodimerization and protein-protein interactions ${ }^{14}$. Drosophila expresses five different heterochromatin protein family members ${ }^{12}$ termed HP1a-HP1e. HP1a (hereafter termed as HP1, encoded by Su(var)2-5) was discovered in Drosophila ${ }^{15}$ and is essential for early embryonic development, as is the mammalian protein HP1 $\beta^{16,17}$. HP1 localizes mainly to $\mathrm{H} 3 \mathrm{~K} 9 \mathrm{me} 2 / 3$-rich heterochromatin ${ }^{10,15,18}$, but also to euchromatic sites along chromosome arms ${ }^{19}$. HP1 might promote heterochromatin compaction through phase separation ${ }^{20}$, similar to human $\mathrm{HP} 1 \alpha^{21}$. Whether HP1 is required to initiate genome reorganization in early embryos is unclear.

To address this question, we performed immunofluorescence of Drosophila embryos before ZGA and the establishment of higher-order chromatin architecture ${ }^{5,6}$, observing diffuse nuclear localization of HP1 (Fig. 1a, Extended Data Fig. 1a). By ZGA, both HP1 and H3K9me3 were strongly enriched at pericentromeric heterochromatin, which was localized apically (reflecting the Rabl configuration) and overlapped with DAPI-dense regions (Fig. 1b, Extended Data Fig. 1b, c). The HP1 signal was around 30 times higher in these regions (Supplementary Methods).

To characterize HP1 binding at different developmental stages, we performed HP1ChIP-seq in precisely hand-staged Drosophila wild-type (control) embryos (Fig. 1c, Extended Data Fig.1d, e). At ZGA, HP1 localized not only to constitutive heterochromatin, such as pericentromeric and telomeric regions (4,394 peaks, 67\%) (Extended Data Fig. 1d), but also within chromosome arms $(2,213$ peaks, $33 \%)$ at repeat sequences (43\% of non-pericentromeric peaks, $10 \%$ long interspersed nuclear elements (LINEs), 30\% long-terminal repeats (LTRs)) and unique sequences ( $57 \%$ of peaks) (Extended Data Fig. 1d-g). Consistent with the immunofluorescence analysis (Fig. 1a), HP1 was bound to chromatin even in totipotent nuclei (Fig. 1c-e), albeit at a lower enrichment ( $16 \%$ of the ZGA enrichments) (Supplementary Methods). Notably, the peak size on chromosome arms did not change markedly (Fig.1d), whereas HP1 spreading occurred at pericentromeric regions during development (Fig. 1e, Extended Data Fig. 1d, Supplementary Methods).

Next, we generated $\mathrm{Hi}-\mathrm{C}$ data for control embryos precisely hand-staged at ZGA (Fig. 2a, Extended Data Fig. 2a). Chromosomes were clearly segregated into A- and B-compartments(Fig. 2a,b). HP1 was bound not only within B-compartment but also within A-compartment

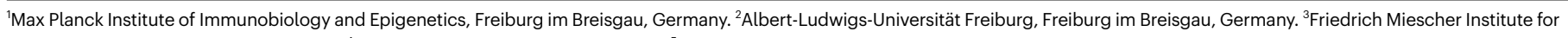
Biomedical Research, Basel, Switzerland. ${ }^{4}$ University of Basel, Basel, Switzerland. ${ }^{5} \mathrm{CIBSS}$ - Centre for Integrative Biological Signalling Studies, University of Freiburg, Freiburg im Breisgau, Germany. ${ }^{6}$ Università degli Studi di Milano and INFN, Milan, Italy. ${ }^{7}$ These authors contributed equally: Fides Zenk, Yinxiu Zhan. ${ }^{凶}$ e-mail: luca.giorgetti@fmi.ch; iovino@ie-freiburg.mpg.de 
a
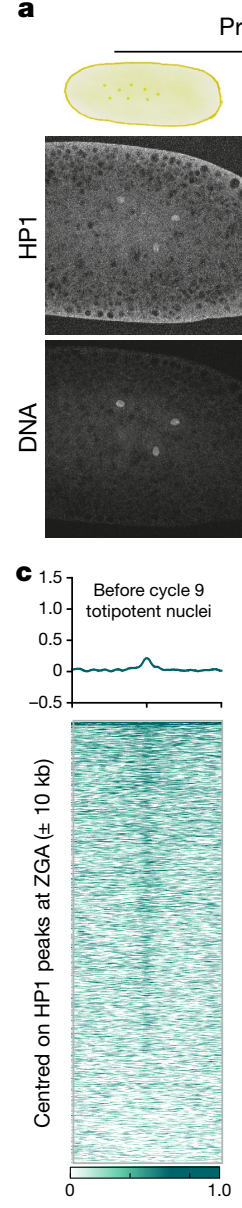

Pre-ZGA
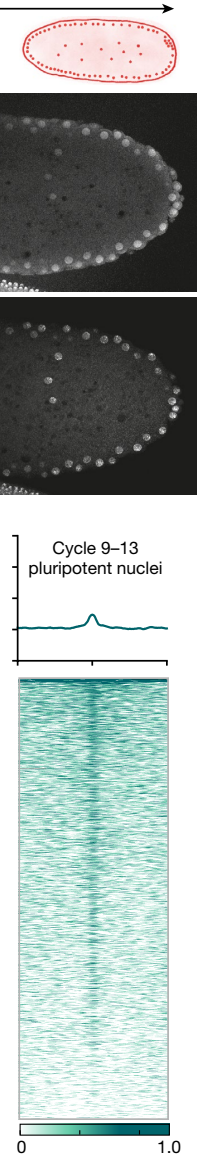
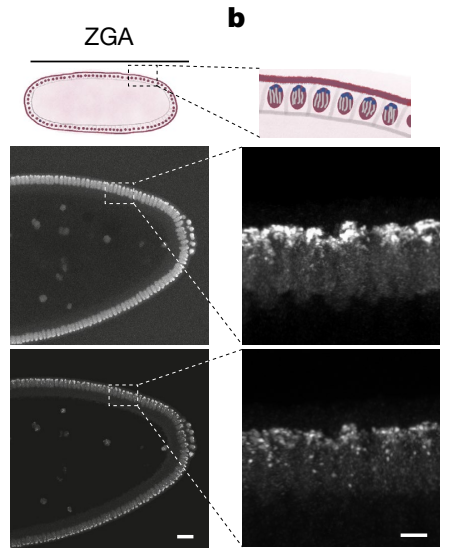
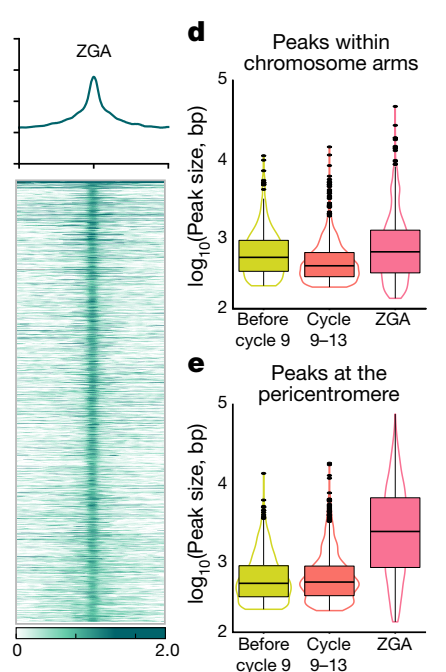

Fig. 1 | Localization of HP1 during early embryonic development. a, Top, schematic of early embryonic development. Bottom, immunofluorescence staining at different stages of early embryonic development. HP1 localizes to chromatin before ZGA and becomes enriched the pericentromeric heterochromatin at ZGA. Scale bar, $20 \mu \mathrm{m}$. b. Close-up view of HP1 localization at ZGA. Top, schematic shows the Rabl configuration of the chromosomes at this developmental stage, with the centromeres localizing on top and the chromosome arms reaching to the bottom of the nucleus. Bottom, the centromeric regions display strong HP1 signals. Images in $\mathbf{a}$ and $\mathbf{b}$ are representative from four biological replicates. Scale bar, $5 \mu \mathrm{m}$.c. Heat maps of HP1 ChIP-seq signal at three different early embryonic developmental time points. The signal is centred on HP1 peaks within chromosome arms called at ZGA and ranked by signal intensity at cycles $9-13$. HP1 binding to chromatin is already observed before cycle 9 , and becomes more enriched during development. d, Box plots of HP1 peak size distribution within chromosome arms at cycle 9, cycles 9-13 and ZGA.e, Box plots of HP1 peak size distribution within pericentromeric regions at cycle 9, cycles 9-13 and ZGA, showing that HP1 peaks get broader at the pericentromeric regions at ZGA. In all box plots, centre line denotes the median; boxes denote lower and upper quartiles (Q1 and Q3, respectively); whiskers denote $1.5 \times$ the interquartile region (IQR) below Q1 and above Q3; points denote outliers.

sequences (Fig. 2c, d, Extended Data Fig. 2b-d, Supplementary Methods). As expected, HP1 binding in B-compartment regions systematically overlapped with $\mathrm{H} 3 \mathrm{~K} 9 \mathrm{me} 3$, localized around repeats and occasionally extended over several kilobases (median peak size 730 bp) (Fig. 2c). By contrast, we detected two different modes of HP1 binding in A-compartment regions. We found that $46 \%$ of HP1 binding sites in the A-compartment were sharply localized and enriched for active chromatin marks, and did not overlap with repeats (Fig. 2d, Extended Data Fig. 2d, cluster 2). A second class of HP1 peaks resembled those in the B-compartment (Extended Data Fig. 2d, cluster 1). These

might correspond to short stretches of repetitive repressed DNA that cannot be resolved unequivocally by $\mathrm{Hi}-\mathrm{C}$. ChIP-seq analysis thus suggests that HP1 binds (1) within active, H3K9ac-rich chromatin in the A-compartment, and (2) within inactive, constitutive heterochromatic domains of the B-compartment.

To explore the role of HP1 in establishing 3D chromosome organization, we examined early embryos that were depleted of maternally supplied HP1. Because HP1 is essential in Drosophila ${ }^{15}$, we performed conditional knockdown ${ }^{22}$ (Extended Data Fig. 3a, Supplementary Methods).

Complete depletion of HP1 blocked development before ZGA, whereas partial knockdown of HP1 still supported development to ZGA (Extended Data Fig. 3b, c, Supplementary Methods). Therefore, we used the partial HP1-knockdown (HP1-KD) embryos in all subsequent experiments. The embryonic lethality of the partial HP1-KD embryos was rescued with a short hairpin RNA (shRNA)-resistant HP1 (HP1-rescue) (Extended Data Fig. 3d), confirming the specificity. HP1 depletion led to strongly reduced binding of HP1 genome-wide, and to upregulation of the telomeric retroelement Het-A that was rescued in HP1-rescue embryos (Extended Data Figs.1g, 3e, f).

$\mathrm{Hi}-\mathrm{C}$ analysis of HP1-KD embryos at ZGA revealed major genome-wide changes in chromosome organization (Fig. 3a, Extended Data Fig.3g, h); we found perturbed Rabl configuration with decreased contact frequencies within and between pericentromeric regions and reduced inter-arm and inter-chromosomal contacts (Fig. 3a). Unexpectedly, we also observed increased intra-chromosomal contacts and milder decay of contact probabilities within chromosome arms (Fig. 3b-d), which suggests an overall increase in chromosome compaction within arms.

Notably, HP1-KD embryos also showed reduced segregation of A- and B-compartments, with a $20 \%$ decrease in B-compartment strength (Fig. 3e, Extended Data Fig. 3i,j). This effect was consistent across replicates, chromosome arms and for inter-arm and inter-chromosome contacts (Extended Data Fig. 3j, k). We found almost no compartment switching (Extended Data Fig. 3l). We also detected decreased insulation across topologically associating domains (TADs) (Extended Data Fig. $3 \mathrm{~m}, \mathrm{n}$ ). By excluding short-range contacts (less than $500 \mathrm{~kb}$ or $3 \mathrm{Mb}$ ), we confirmed that the reduction of the B-compartment signal is independent of the reduction in TAD insulation (Extended Data Fig. 30). Crucially, all of these phenotypes were rescued in HP1-rescue embryos (Extended Data Fig. 4a-d).

To validate the structural defects observed in HP1-KD embryos by $\mathrm{Hi}-\mathrm{C}$ analysis, we performed 3D DNA fluorescence in situ hybridization (3D DNA FISH) with oligonucleotide probes spanning several megabases on chromosomes $2 \mathrm{R}$ and $3 \mathrm{~L}$ (Fig. 3f, g). Quantitative image analysis of single cells showed that chromosomes were on average separated by larger distances (around 30\% increase) in HP1-KD embryos (Fig. 3h, Supplementary Methods), in line with reduced inter-arm and inter-chromosome interactions observed in $\mathrm{Hi}$-C data (Fig. 3a). In agreement with Hi-C data (Fig. 3b), we also found that the volume of the FISH signals was significantly decreased (around 10\% decrease) (Supplementary Methods) in HP1-KD embryos (Fig. 3i), which suggests increased compaction of chromosome arms.

HP1 depletion thus perturbs the overall nuclear structure, with reduced proximity between pericentromeric regions, reduced alignment of chromosome arms and increased intra-chromosomal compaction. These global effects are accompanied by a prominent loss of contacts within B-compartment regions. The structural defects of HP1-KD embryos are notable, given that depletion of HP1 was only partial to allow embryos to reach ZGA. Our findings reveal that HP1 has a key role in establishing the 3D genome structure during development.

Only a small fraction of genes and repeats was misregulated in HP1-KD embryos at ZGA (Extended Data Fig. 4e). The most highly upregulated retroelements were localized at telomeric regions (Het-A, TAHRE and TART retrotransposons) and cannot account for the structural changes that we observed genome-wide(Extended Data Fig. 4e,f). We confirmed 

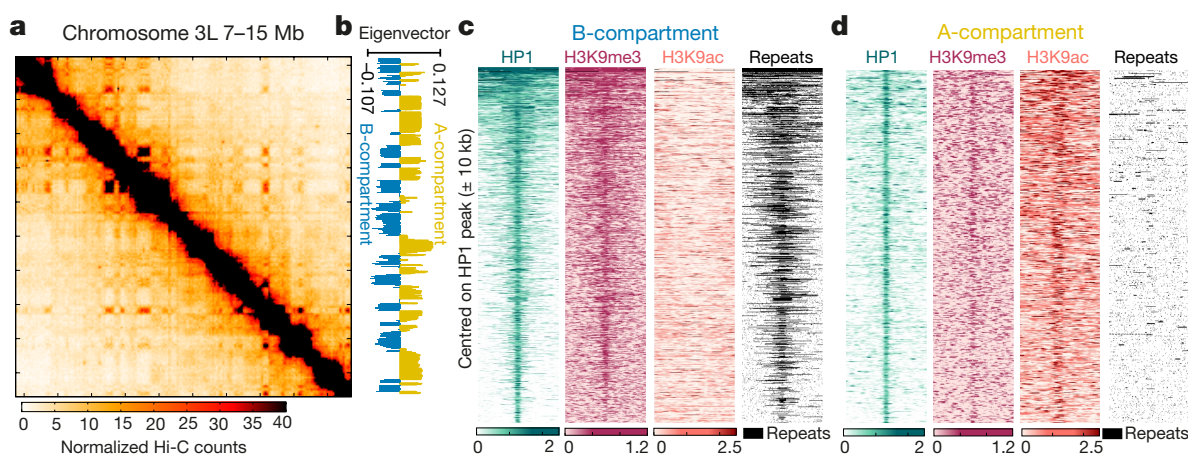

Fig. 2 HP1 binds both A- and B-compartment regions at ZGA. a, Hi-C contact map of an 8-Mb region on chromosome $3 \mathrm{~L}$ (resolution $40 \mathrm{~kb}$ ). Pooled $\mathrm{Hi}-\mathrm{C}$ data of seven biological replicates are shown (Extended Data Fig. 2a). b, Compartment scores (first eigenvector of the $\mathrm{Hi}-\mathrm{C}$ map, resolution: $10 \mathrm{~kb}$ ), same region as in a (Supplementary Methods).c, Heat maps of HP1, H3K9me3

and $\mathrm{H} 3 \mathrm{~K}$ 9ac ChIP-seq signals as well as repeat positions, $\pm 10 \mathrm{~kb}$ centred on HP1 peaks occurring in B-compartment regions. HP1 binding overlaps with broad $\mathrm{H} 3 \mathrm{~K} 9 \mathrm{me} 3$ peaks, repeats and is devoid of $\mathrm{H} 3 \mathrm{~K} 9 \mathrm{ac}$. d, As in $\mathbf{c}$ for $\mathrm{HP} 1$ peaks in A-compartment regions, showing enrichment in $\mathrm{H} 3 \mathrm{~K} 9 \mathrm{ac}$ and absence of repeats (Extended Data Fig. 2b-d).

that HP1-KD embryos did not show defects in the onset of transcription at ZGA, and that both the control and the HP1-KD embryos at ZGA were in interphase (Extended Data Fig. 4g, h).

To investigate the role of HP1 in the establishment versus the maintenance of chromatin structures, we performed $\mathrm{Hi}$-C experiments with differentiated, somatic Drosophila S2 cells. Notably, HP1 depletion did

not considerably affect genome architecture (Extended Data Fig. 4i-o), which suggests that HP1 is not required to maintain chromatin structure.

Because HP1 interacts with chromatin by binding to $\mathrm{H} 3 \mathrm{~K} 9 \mathrm{me} 2 / 3$, we generated embryos depleted of $\mathrm{H} 3 \mathrm{~K} 9 \mathrm{me} 2 / 3$ by overexpressing the histone 3 lysine 9-to-methionine (H3K9M) mutation ${ }^{23}$ (Extended
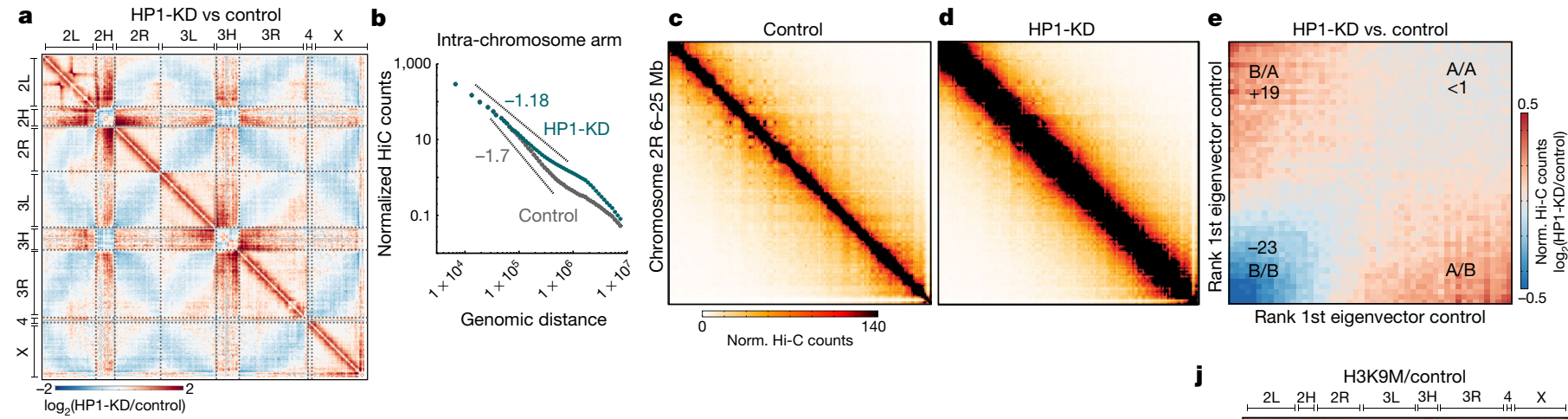
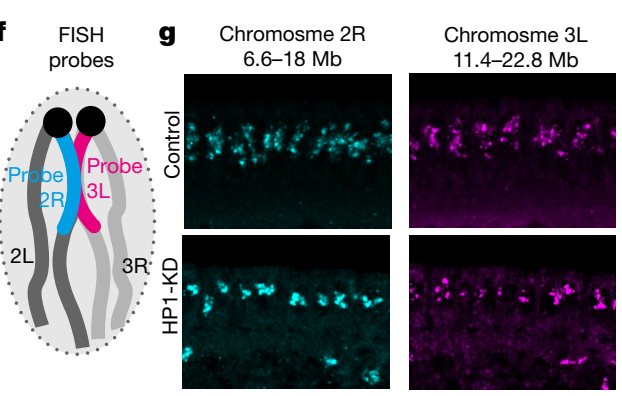

$\mathrm{DAPI}+$ merge

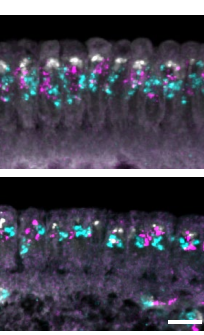

Fig. 3 Depletion of HP1 causes increased intra-chromosome compaction and reduced compartmentalization. a, Differential $\mathrm{Hi}-\mathrm{C}$ contact map ( $\log _{2}$-transformed), highlighting increased contact frequencies within chromosome arms, decreased inter-arm and inter-chromosome contacts, reduced associations within and between pericentromeric regions, and increased interactions of pericentromeric regions with chromosome arms in HP1-KD embryos. Biological replicates were pooled; $n=7$ control and $n=5$ HP1-KD embryos. b, HP1-KD embryos show a milder decay of contact probabilities above $100 \mathrm{~kb}$. c, Hi-C contact maps of $19 \mathrm{Mb}$ on chromosome 2R in control embryos (resolution: $120 \mathrm{~kb}$ ). d, As in c, in HP1-KD embryos. e, Differential contact enrichment in HP1-KD versus control embryos, sorted by compartment score (Supplementary Methods), shows decreased B-compartment interactions and increased A/B intermixing. Changes relative to the control.f, Scheme of FISH probe design to quantify inter-arm distance
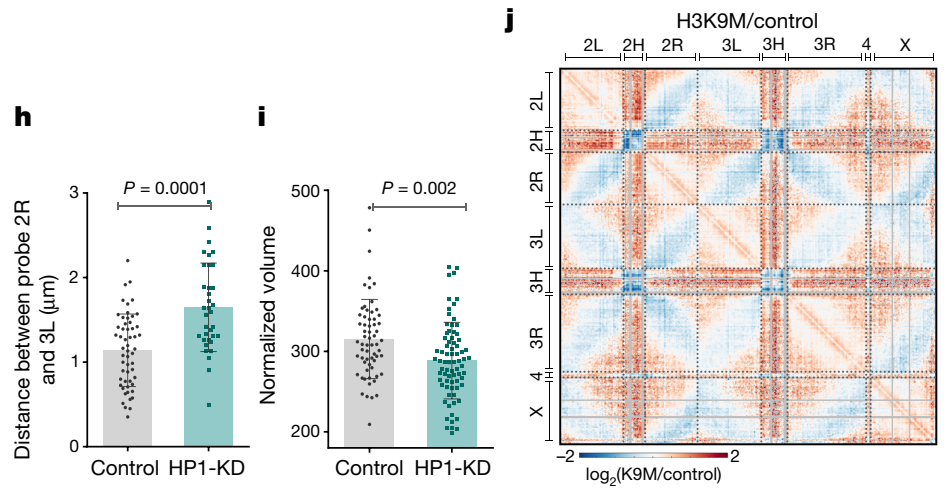

and intra-arm compaction. g, Representative 3D-DNA FISH staining of control and HP1-KD embryos at ZGA. Signals from probes on chromosome 2 R and chromosome $3 \mathrm{~L}$ are shown separately and merged with DAPI staining. Scale bar, $5 \mu \mathrm{m}$. h, Quantification of physical distances between FISH signals from chromosome $2 \mathrm{R}$ and $3 \mathrm{~L}$ (mean \pm s.d., nuclei: control $n=55, \mathrm{HP} 1-\mathrm{KD} n=35$ ). i, Quantification of compaction of FISH signals from chromosome $2 \mathrm{R}$ (mean \pm s.d., nuclei: control $n=63$, HP1-KD $n=75$ ).j, Differential Hi-C contact map ( $\log _{2}$-transformed), highlighting decreased inter-arm and inter-chromosomal contacts, reduced associations within and between pericentromeric regions, and increased interactions of pericentromeric regions with chromosome arms in $\mathrm{H} 3 \mathrm{~K} 9 \mathrm{M}$ embryos. Biological replicates were pooled; $n=7$ control and $n=2 \mathrm{H} 3 \mathrm{~K} 9 \mathrm{M}$ embryos. See Supplementary Methods and Extended Data Fig. 5 for further details. $P$ values were determined by Wilcoxon two-sided test. 

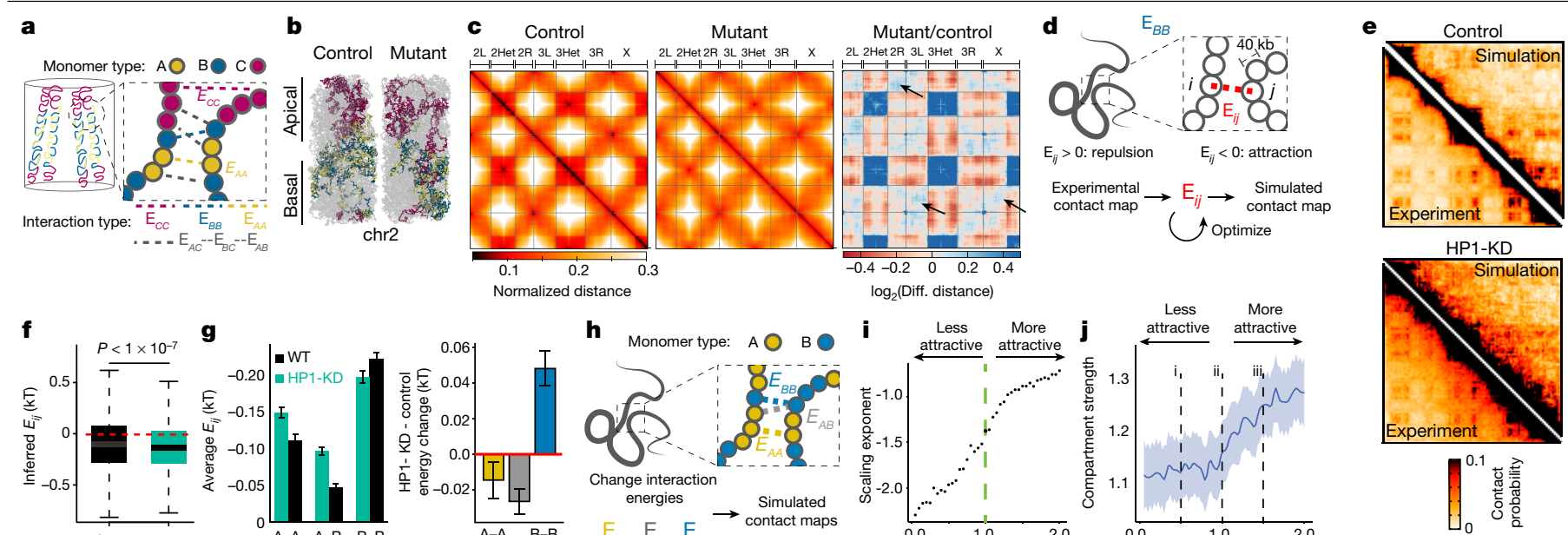
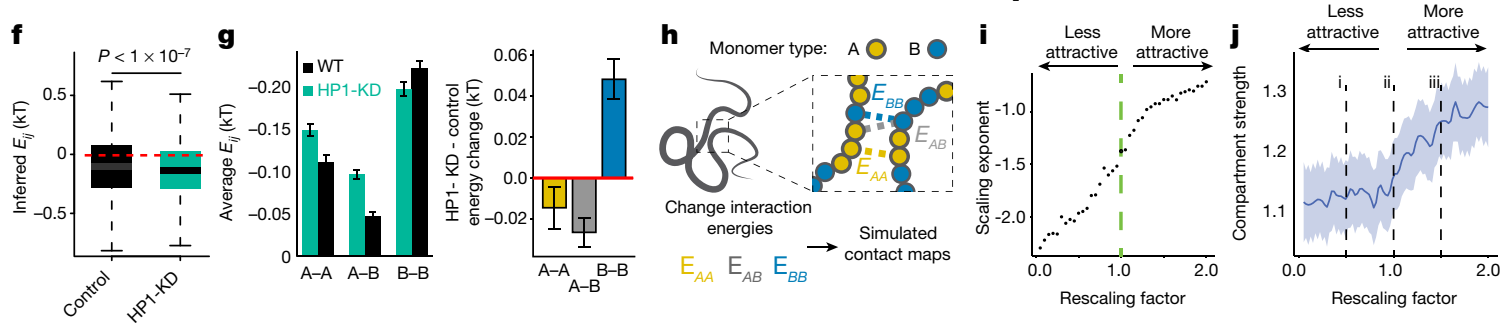

determined by two-sided Wilcoxon test. Box plots are as in Fig. 1d. g, Left, interaction energies between $B$-type beads $(B-B)$ become comparatively less attractive in HP1-KD embryos, but more attractive between A-type beads (A-A) and between $A$ and $B$ types ( $A-B)$. Right, average interaction energy changes between HP1-KD and control models. B-compartment attractions decrease in the HP1-KD model. Data are mean \pm s.e.m., interactions: 990 (A-A), 2,069 (A-B), $1,035(B-B) . h$, Chromatin is modelled as a chain of two types ( $A$ and $B$ ) of interacting 40-kb beads (chr3R 17-20.6 Mb). i, Scaling exponents increase when attractions between all beads are increased by a multiplicative factor, and vice versa.j, Compartment strength (bold line: mean) decreases when attractions between beads are increased, and vice versa. Confidence interval (shaded area) calculated using t-based approximation.
Data Fig. 5a). Quantitative ChIP-seq for HP1 in precisely hand-staged H3K9M embryos at ZGA showed that HP1 binding was greatly reduced on pericentromeric and repeat regions as well as chromosome arms (Extended Data Fig. 5b-d). However, HP1 was 20\% more retained on chromosome arms in H3K9M compared to HP1-KD embryos (Extended Data Fig. 5b, right), which could be due to some residual H3K9me2/3 and/or H3K9me2/3-independent binding of HP1 (Extended Data Fig. 5d, right, cluster 2). ChIP-seq analysis of chromodomain-mutant HP1 (HP1-CD) ${ }^{13}$ also revealed some residual binding on chromosome arms, further supporting H3K9me2/3-independent binding of HP1 (Extended Data Fig. 5e).

$\mathrm{Hi}-\mathrm{C}$ maps of $\mathrm{H} 3 \mathrm{~K} 9 \mathrm{M}$ embryos revealed pericentromeric heterochromatin de-clustering and reduced chromosome arm alignment, but only a mild gain in chromosome arm compaction and mild defects in compartmentalization (Fig. $3 \mathrm{j}$, Extended Data Fig. $5 f-j$ ), which could be explained by higher retention of HP1 along chromosome arms in H3K9M embryos (Extended Data Fig. 5b).

Overall, our data indicate that HP1 has a major role in establishing chromatin architecture in early embryos by: (1) mediating the clustering and condensation of constitutive heterochromatin at pericentromeric regions through $\mathrm{H} 3 \mathrm{~K} 9 \mathrm{me} 2 / 3$-dependent binding; (2) aiding the overall configuration of chromosome arms; and (3) contributing to the formation of the B-compartment.

Next, we set out to exclude that folding defects observed at chromosome arms in HP1-KD embryos could arise as a mere consequence of the expansion of pericentromeric chromatin. Because it is impossible to completely decouple these effects in vivo, we turned to a genome-wide polymer modelling approach in which chromosomes are represented as chains of three types of $10-\mathrm{kb}$ beads (A, B and C corresponding to A- and B-compartment and pericentromeric/telomeric regions, respectively) confined in a cylindrical nucleus (Fig. 4a, Supplementary Methods). We first optimized a set of interaction energies to reproduce contact probability scaling and compartment strength within arms in control embryos (Extended Data Fig. 6a-c). Next, we mimicked centromere de-clustering by decreasing interactions among $C$-type beads and their interactions with the nuclear surface (mutant) (Fig. 4b, c). The model recapitulated reduced alignment between chromosome arms (Fig. 4c, right) and increased interactions between pericentromeric regions and chromosome arms (Fig. 4c), but not compaction and compartmentalization defects within arms (Extended Data Fig. 6d, e). These results do not depend on the numbers of centromeric and telomeric beads (Extended Data Fig. 6f-l). This suggests that compartment defects and intra-arm compaction are a consequence of decreased HP1 binding on chromosome arms.

To understand the cause of compartment defects in HP1-KD embryos and determine whether they might simply arise from increased intra-arm compaction (Fig. 3a-d), we implemented two smaller-scale polymer models designed to uncover the energies driving the folding of chromosome arms.

In the first approach, interaction energies between $40-\mathrm{kb}$ beads were optimized to reproduce experimental $\mathrm{Hi}-\mathrm{C}$ maps within multi-megabase regions of chromosome arms ${ }^{24,25}$ (Fig. 4d, Supplementary Methods). For control contact maps (Fig. 4e, top), we found that interaction energies were globally attractive, which accounts for the correct contact probability scaling (Extended Data Fig. 7a), The model predicted that $\mathrm{A}-\mathrm{A}$ and $\mathrm{B}-\mathrm{B}$ interactions were on average more attractive than A-B interactions (Extended Data Fig. 7b). For HP1-KD contact maps (Fig. 4e, bottom, Extended Data Fig. 7c, d), we found increased attractions overall between all bead types but comparatively less attractive B-B interactions (Fig. 4f, g). Notably, our findings do not depend on the specific region that is simulated (Extended Data Fig. 7e-I). This suggests that decreased compartmentalization is not a mere consequence of increased compaction after HP1 knockdown (Fig. 3a-d) but instead requires the simultaneous loss of $\mathrm{B}$-specific attractive interactions. 
To confirm these findings, we used a more general model that is not designed to reproduce the experimental $\mathrm{Hi}-\mathrm{C}$ maps but instead describes the behaviour of a polymer when interaction energies between its constituent $A$ - and $B$-type beads are systematically varied (Fig. 4h, Supplementary Methods). Increasing all A-A, A-B and B-B interaction energies correctly predicted milder scaling of contact probabilities (such as HP1-KD), but led to stronger compartments (Fig. 4i,j, Extended Data Fig. $7 \mathrm{~m}$ ). By contrast, decreasing all interaction energies correctly predicted compartment loss but led to the wrong scaling behaviour (steeper decay) (Fig. 4i, j, Extended Data Fig. 7m). Finally, decreasing only $\mathrm{B}-\mathrm{B}$ attractions reproduced the observed decrease in compartment strength but resulted in a steeper scaling (Extended Data Fig. 7n). Thus, modifying chromosome compaction alone cannot explain the HP1-KD structural phenotype, which suggests that HP1 depletion perturbs compartmental forces. Notably, these results do not depend on the distribution of A- and B-compartment beads (Extended Data Fig. 7o-r). Analysis of this general polymer model shows that the HP1-KD structural phenotype within arms (increased compaction, lower compartmentalization) arises from two independent mechanisms: decreased specific interactions between B-compartment regions, and increased attraction between all genomic locations.

Our data and modelling approaches suggest that HP1-mediated interactions, which might occur through HP1 oligomerization ${ }^{14}$ or phase separation ${ }^{20,21}$, have a major role in establishing 3D genome conformation during embryogenesis. Decreased HP1 binding in pericentromeric heterochromatin led to declustering and decondensation of constitutive heterochromatin and a perturbed Rabl configuration. By contrast, decreased HP1 levels within chromosome arms caused decreased B-B compartment attractions and increased arm compaction, possibly owing to decreased chromatin stiffness. Reduced segregation of B-compartment regions after HP1 knockdown might facilitate interactions between A- and B-type chromatin and allow attractions between active regions to dominate, resulting in globally increased compaction (Extended Data Fig. 7s). This is consistent with quantitative compartment analysis (Fig. 3e, Extended Data Fig. 3i, j) and the overall increase in $\mathrm{A}-\mathrm{A}$ and $\mathrm{A}-\mathrm{B}$ interactions in simulations (Fig. 4g). Alternatively, increased attractions could arise from HP1 counteracting condensin II-mediated homologous chromosome pairing or cohesin-mediated loop extrusion.

In the A-compartment, HP1-mediated compartmental forces might be counteracted by surrounding active chromatin modifications such as $\mathrm{H} 3 \mathrm{~K}$ 9ac (Fig. 2d, Extended Data Fig. $7 \mathrm{~s}$ ). Because the A-compartment is not affected after disruption of the B-compartment (Fig. 3e), we suggest that it is controlled by a distinct driving force independent of HP1.

Our study shows that HP1 is required to establish pericentromeric heterochromatin clustering in early embryos but is dispensable in differentiated cells, consistent with a recent report in mammals ${ }^{26}$. In differentiated cells, clustering might be driven by other HP1 paralogues or heterochromatin proteins ${ }^{2}$ favoured by the slower cell cycle, or result from other mechanisms involving solid-like states in heterochromatin condensates ${ }^{27}$. We also showed that HP1 prevents the collapse of chromosome arms while they elongate to establish the characteristic Rabl configuration. Finally, HP1 is directly involved in the formation of the $\mathrm{B}$ - but not the A-compartment region. Because pericentromeric clustering and compartmentalization also occur in mammals, HP1 could have similar functions during mammalian embryogenesis.

\section{Online content}

Any methods, additional references, Nature Research reporting summaries, source data, extended data, supplementary information, acknowledgements, peer review information; details of author contributions and competing interests; and statements of data and code availability are available at https://doi.org/10.1038/s41586-021-03460-z.

1. Falk, M. et al. Heterochromatin drives compartmentalization of inverted and conventional nuclei. Nature 570, 395-399 (2019)

2. Hildebrand, E. M. \& Dekker, J. Mechanisms and functions of chromosome compartmentalization. Trends Biochem. Sci. 45, 385-396 (2020).

3. Marshall, W. F., Dernburg, A. F., Harmon, B., Agard, D. A. \& Sedat, J. W. Specific interactions of chromatin with the nuclear envelope: positional determination within the nucleus in Drosophila melanogaster. Mol. Biol. Cell 7, 825-842 (1996).

4. Merico, V. et al. Epigenomic differentiation in mouse preimplantation nuclei of biparental, parthenote and cloned embryos. Chromosome Res. 15, 341-360 (2007).

5. Hug, C. B., Grimaldi, A. G., Kruse, K. \& Vaquerizas, J. M. Chromatin architecture emerges during zygotic genome activation independent of transcription. Cell 169, 216-228 (2017).

6. Ogiyama, Y., Schuettengruber, B., Papadopoulos, G. L., Chang, J. M. \& Cavalli, G.

Polycomb-dependent chromatin looping contributes to gene silencing during Drosophila development. Mol. Cell 71, 73-88 (2018).

7. Du, Z. et al. Allelic reprogramming of 3D chromatin architecture during early mammalian development. Nature 547, 232-235 (2017).

8. Collombet, S. et al. Parental-to-embryo switch of chromosome organization in early embryogenesis. Nature 580, 142-146 (2020)

9. Lieberman-Aiden, E. et al. Comprehensive mapping of long-range interactions reveals folding principles of the human genome. Science 326, 289-293 (2009).

10. Fodor, B. D., Shukeir, N., Reuter, G. \& Jenuwein, T. Mammalian Su(var) genes in chromatin control. Annu. Rev. Cell Dev. Biol. 26, 471-501 (2010).

11. Allshire, R. C. \& Madhani, H. D. Ten principles of heterochromatin formation and function. Nat. Rev. Mol. Cell Biol. 19, 229-244 (2018).

12. Vermaak, D. \& Malik, H. S. Multiple roles for heterochromatin protein 1 genes in Drosophila. Annu. Rev. Genet. 43, 467-492 (2009).

13. Lachner, M., O'Carroll, D., Rea, S., Mechtler, K. \& Jenuwein, T. Methylation of histone H3 lysine 9 creates a binding site for HP1 proteins. Nature 410, 116-120 (2001).

14. Machida, S. et al. Structural basis of heterochromatin formation by human HP1. Mol. Cell 69, 385-397 (2018).

15. James, T. C. \& Elgin, S. C. Identification of a nonhistone chromosomal protein associated with heterochromatin in Drosophila melanogaster and its gene. Mol. Cell. Biol. 6, 3862-3872 (1986)

16. Aucott, R. et al. HP1-beta is required for development of the cerebral neocortex and neuromuscular junctions. J. Cell Biol. 183, 597-606 (2008).

17. Santos, F., Peters, A. H., Otte, A. P., Reik, W. \& Dean, W. Dynamic chromatin modifications characterise the first cell cycle in mouse embryos. Dev. Biol. 280, 225-236 (2005).

18. James, T. C. et al. Distribution patterns of HP1, a heterochromatin-associated nonhistone chromosomal protein of Drosophila. Eur. J. Cell Biol. 50, 170-180 (1989).

19. Marsano, R. M., Giordano, E., Messina, G. \& Dimitri, P. A New Portrait of Constitutive Heterochromatin: Lessons from Drosophila melanogaster. Trends Genet. 35, 615-631 (2019).

20. Strom, A. R. et al. Phase separation drives heterochromatin domain formation. Nature 547, 241-245 (2017).

21. Larson, A. G. et al. Liquid droplet formation by HP1a suggests a role for phase separation in heterochromatin. Nature 547, 236-240 (2017).

22. Zenk, F. et al. Germ line-inherited H3K27me3 restricts enhancer function during maternal-to-zygotic transition. Science 357, 212-216 (2017).

23. Herz, H. M. et al. Histone $\mathrm{H} 3$ lysine-to-methionine mutants as a paradigm to study chromatin signaling. Science 345, 1065-1070 (2014).

24. Giorgetti, L. et al. Predictive polymer modeling reveals coupled fluctuations in chromosome conformation and transcription. Cell 157, 950-963 (2014).

25. Zhan, Y., Giorgetti, L. \& Tiana, G. Modelling genome-wide topological associating domains in mouse embryonic stem cells. Chromosome Res. 25, 5-14 (2017).

26. Erdel, F. et al. Mouse heterochromatin adopts digital compaction states without showing hallmarks of HP1-driven liquid-liquid phase separation. Mol. Cell 78, 236-249 (2020).

27. Strickfaden, $\mathrm{H}$. et al. Condensed chromatin behaves like a solid on the mesoscale in vitro and in living cells. Cell 183, 1772-1784.e13 (2020).

Publisher's note Springer Nature remains neutral with regard to jurisdictional claims in published maps and institutional affiliations.

Open Access This article is licensed under a Creative Commons Attribution 4.0 International License, which permits use, sharing, adaptation, distribution and reproduction in any medium or format, as long as you give appropriate credit to the original author(s) and the source, provide a link to the Creative Commons license, and indicate if changes were made. The images or other third party material in this article are included in the article's Creative Commons license, unless indicated otherwise in a credit line to the material. If material is not included in the article's Creative Commons license and your intended use is not permitted by statutory regulation or exceeds the permitted use, you will need to obtain permission directly from the copyright holder. To view a copy of this license, visit http://creativecommons.org/licenses/by/4.0/.

(c) The Author(s) 2021 


\section{Article}

\section{Reporting summary}

Further information on research design is available in the Nature Research Reporting Summary linked to this paper.

\section{Data availability}

All Hi-C, ChIP-seq and RNA sequencing raw files generated in this study have been uploaded to the Gene Expression Omnnibus (GEO) under accession GSE140542. The following public databases were used: BSgenome.Dmelanogaster.UCSC.dm6, org.Dm.eg.db and TxDb.Dmelanogaster.UCSC.dm6.ensGene.Source data are provided with this paper.

\section{Code availability}

Custom code generated in this study is available at: https://github. com/zhanyinx/Zenk_Zhan_et_al_Nature2021.

Acknowledgements We thank the lovino laboratory, in particular R. Schiavo, D. Ibarra Morales and E. Ponzo; T. Kulkarni, A. Panhale and M. Samata from the Akhtar department; A. Andersen, A. Akhtar, T. Boehm, R. Paro, R. Sawarkar and M. Wiese for crucial reading of the manuscript and discussion; T. Jenuwein, G. Reuter and P. Dimitri for the lengthy and insightful discussion about heterochromatin; The Bioinformatics and Sequencing facilities at the MPI-IE; T. Manke, L. Arrigoni and in particular D. Ryan, M. Rauer, L. Rabbani and

G. Renschler. M. Stadler for discussions on data analysis; The Imaging facility, Proteomics facility and Fly facility at the MPI-IE. We thank The Bloomington Drosophila Stock Center (NIH P4OOD018537) and the TRiP at Harvard Medical School (NIH/NIGMS R01-GM084947) for providing fly stocks and DSHB (HP1) for antibodies; G. Pyrowolakis for initial help in designing overexpression and deletion fly lines; A. Akhtar (Rpb3), C. Margulis (Rpb3) G. Reuter (HP1) and S. Heidmann (Rad21, SMC1) for providing antibodies. NIBR computing resources, D. Flanders and E. Tagliavini for help with cluster and server supports. F.Z., M.S. and E.L. are supported by the Max Planck Society and IMPRS program. N.A. was supported by the DFG (German Research Foundation) under Germany's Excellence Strategy (EXC-2189) Project ID: 390939984 . N.I. is supported from the Max Planck Society; Deutsche Forschungsgemeinschaft - Project ID 192904750 - CRC 992 Medical Epigenetics; Behrens-Weise Stiftung; EMBO YIP; CIBSS EXC-2189. This project has also received funding from the European Research Council (ERC) under the European Union's Horizon 2020 research and innovation programme (grant agreement no. 819941) ERC CoG, EpiRIME. Research in the Giorgetti laboratory is supported by the Novartis Research Foundation and the European Research Council (ERC) under the European Union's Horizon 2020 research and innovation (grant agreement no. 759366, BioMeTre).

Author contributions F.Z. performed all the experimental work and initial computational analysis; Y.Z. performed all the computational analysis. P.K. contributed and optimized the genome wide simulation. G.T. contributed to experimental design and data interpretation concerning data simulation. E.L. contributed to microscopy data collection and optimized the 3D FISH protocol. N.A. contributed to fly genetics, immunofluorescence staining and sample collection. M.S. helped in sample collection. N.I. and F.Z. conceived the project. N.I. and L.G. designed and supervised the project with inputs from F.Z. and Y.Z. F.Z., Y.Z., L.G. and N.I. wrote the manuscript.

Funding Open access funding provided by Max Planck Society.

Competing interests The authors declare no competing interests.

Additional information

Supplementary information The online version contains supplementary material available at https://doi.org/10.1038/s41586-021-03460-z.

Correspondence and requests for materials should be addressed to $L$. or N.

Peer review information Nature thanks Leonid Mirny and the other, anonymous, reviewer(s) for their contribution to the peer review of this work.

Reprints and permissions information is available at http://www.nature.com/reprints. 
time [min]

mitotic cycle

migration of

the nuclei

Establishment of 3D conformation

establishment of the epigenetic landscape

recruitment of $\mathrm{HP}$

onset of zygotic

transcription

early embryonic development rapid mitotic divisions genome activation

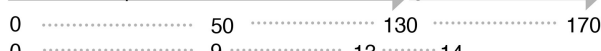

0 ……..................

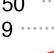

13
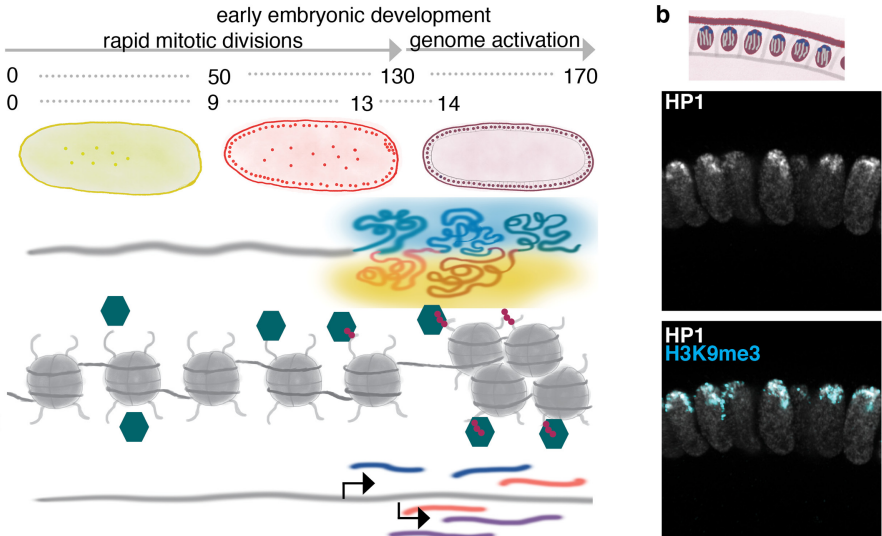

colocalization of HP1 and H3K9me3 in DAPI dense pericentromere
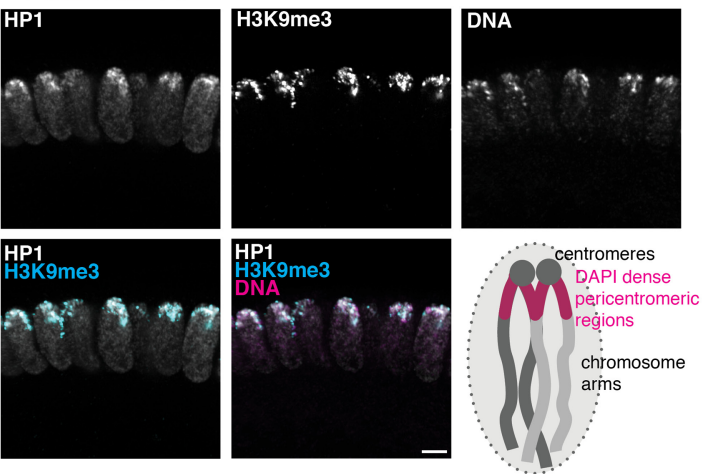

d $\quad$ Chr 2R (1 Mb)

ZGA (0-4 hrs) late embryos (0-14 hrs)
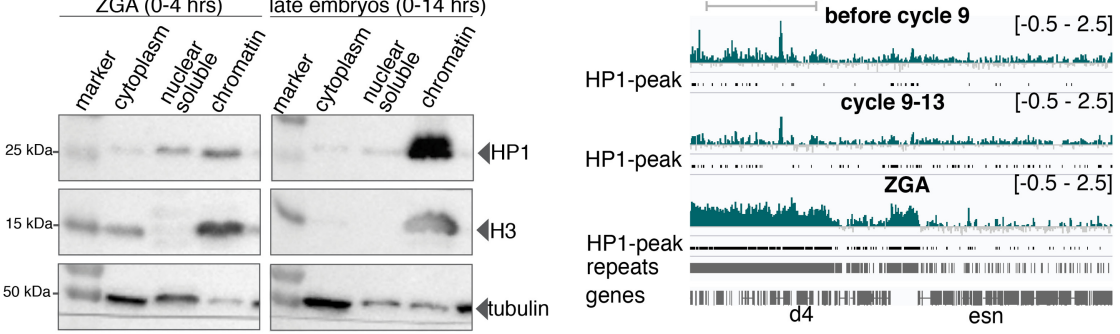

HP1-peak

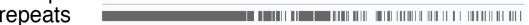

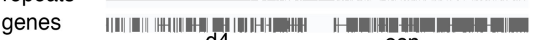

Chr 3R (10 kb)

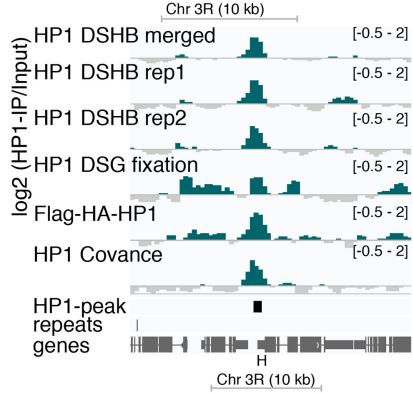

HP1 DSHB merged $\quad[-0.5-2]$

늘HP1 DSHB rep1

HP1 DSHB rep2 - - -

足HP1 DSG fixation

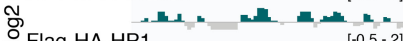

HP1 Covance

HP1-peak $\ldots$ - I H

HP1-peak

genes

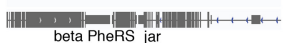

$\mathrm{Chr} 3 \mathrm{R}(10 \mathrm{~kb})$

HP1 DSHB merged $[-0.5-$

늘HP1 DSHB rep1 $-{ }^{[-0.5-2]}$

HP1 DSHB rep2 - - i-0.5.2]

HP1 DSHB rep2

I HP1 DSG fixation [-0.5-2]

胥 Flag-HA-HP1

HP1 Covance $-[-0.5-2]$

HP1 Covance
HP1-peak
repeats
genes

g

g

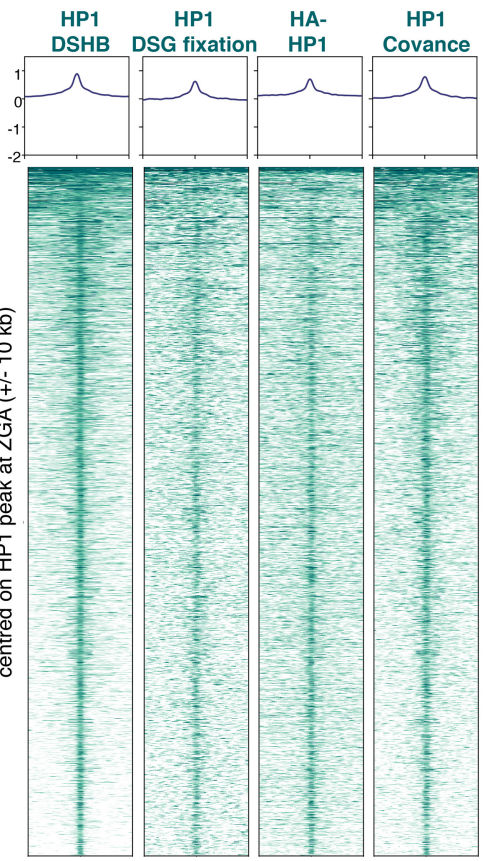

$\longrightarrow 2.0$ e $\operatorname{Chr} 2 \mathrm{R}(20 \mathrm{~kb})$
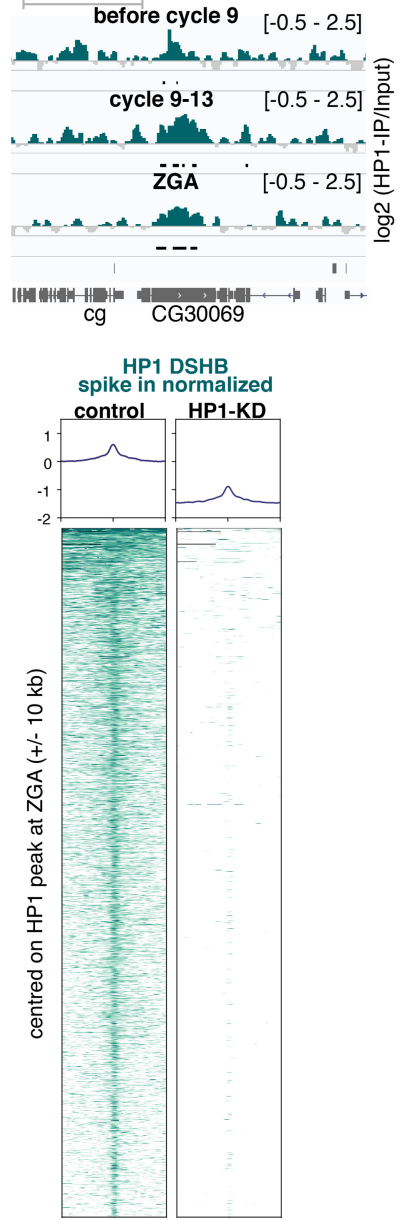

$\longrightarrow 2.0$

Extended Data Fig. 1 | See next page for caption. 


\section{Article}

Extended Data Fig. 1 Characterization of HP1 binding during early embryonic development. a, Cartoon of early Drosophila developmental timing showing the onset of genome organization, chromatin modifications and transcription. $\mathbf{b}$, Immunofluorescence staining of an embryo at ZGA. $\mathrm{H} 3 \mathrm{~K} 9 \mathrm{me} 3$ and $\mathrm{HP} 1$ are enriched at the pericentromeric heterochromatin (clustering on top and corresponding to the DAPI dense signal; see cartoon). Representative image from four biological replicates. Scale bar, $5 \mu \mathrm{m}$. Quantification of the immunof luorescence signal shows that HP1 intensity is 30 times higher in the pericentromeric regions (co-localizing with $\mathrm{H} 3 \mathrm{~K} 9 \mathrm{me} 3$ ) than in the rest of the nucleus. Average signal of 300 nuclei from 2 independent experiments.c, Cellular fractionation of embryonic extracts at $0-4 \mathrm{~h}$ (corresponding to ZGA) of development and from late embryos (corresponding to gastrulation and segmentation). HP1 is already detectable in the chromatin fraction at $0-4 \mathrm{~h}$ and becomes further enriched during differentiation. Representative of two independent experiments. For western blot source data, see Supplementary Fig. 1.d, e, Representative genomic regions showing $\mathrm{HP1}$ signal as $\log _{2}$-transformed fold change over the input before cycle 9 , between cycle 9-13 and at ZGA by ChIP-seq. HP1 peaks and repetitive sequences (UCSC RepeatMasker) are represented below. d, Strong enrichment of HP1 close to the pericentromeric heterochromatin. e, One euchromatic HP1binding region. f, IGV browser snapshots of different genomic regions showing HP1 binding in euchromatin regions. We validated the HP1 ChIP-seq by performing replicate experiments with the same antibody from DSHB (rep1 and rep2). All further tracks in this Article show the merged track (top). To further validate our findings, we mapped the binding of HP1 by performing ChIP-seq against a Flag-haemagglutinin (HA)-tagged transgene and used a second commercial antibody (Covance) and detected the same peaks. We also used disuccinimidyl glutarate (DSG) as crosslinking agent to recover more extended regions of HP1 binding and obtained a similar result of HP1 binding. g, Heat maps of HP1, ChIP-seq signals $\pm 10 \mathrm{~kb}$ centred on HP1 peaks occurring along the chromosome arms at ZGA. We validated the binding profiles by performing ChIP-seq against HP1 with different antibodies (DSHB, HA, Covance) and also used the crosslinker DSG (left). To further validate the peaks within chromosome arms, we performed quantitative ChIP-seq in the HP1-KD background, using $\lambda$-DNA spike-in as normalizer. The HP1 signal is strongly reduced at HP1 peaks within chromosome arms at ZGA (right). See Supplementary Methods for further details. 

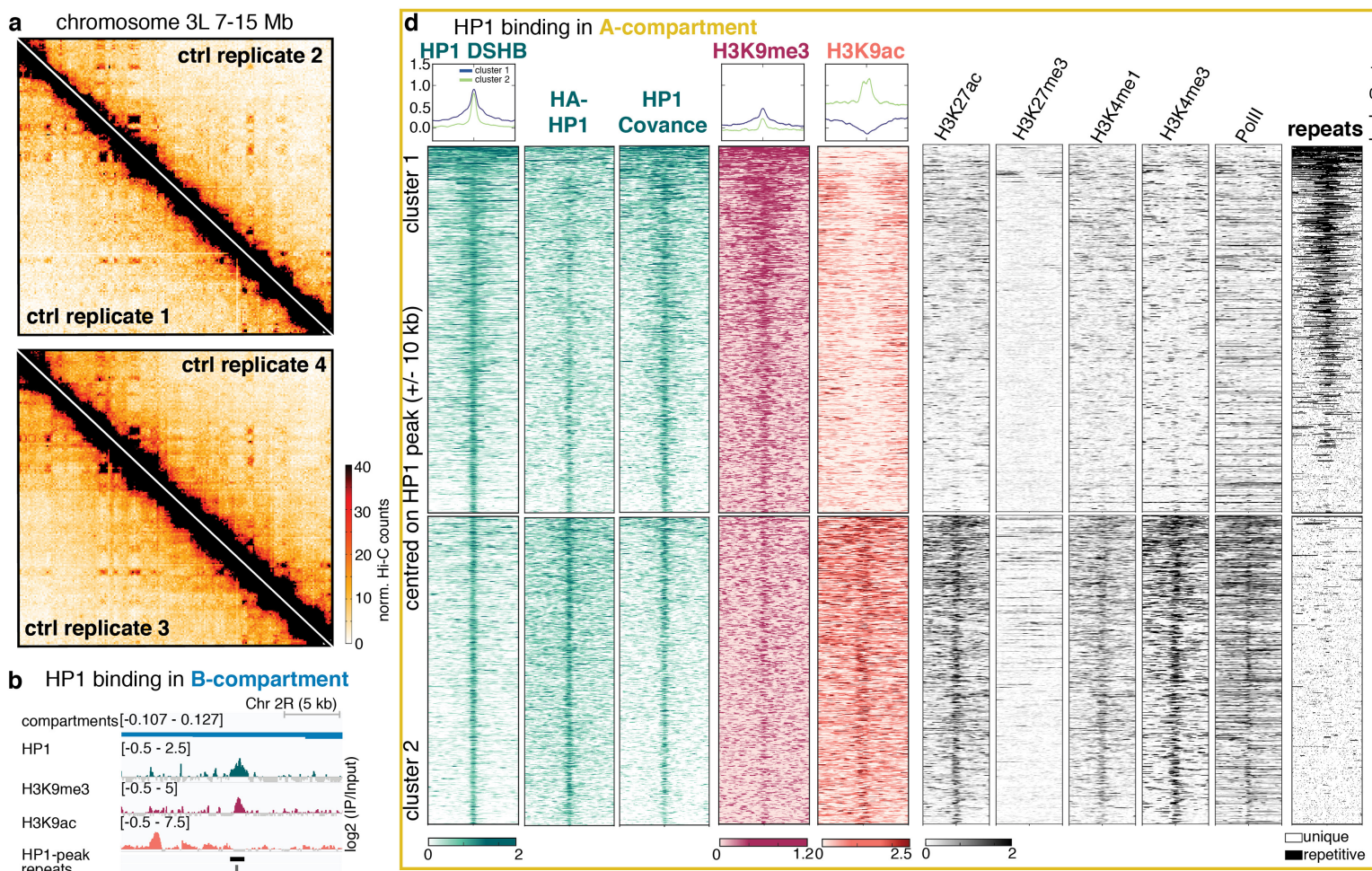

HP1 DSHB
spike in normalized

b HP1 binding in B-compartment

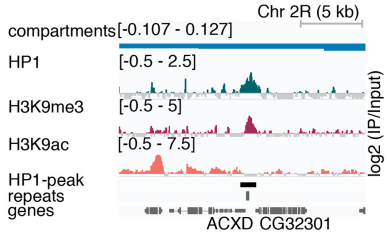

c HP1 binding in A-compartment

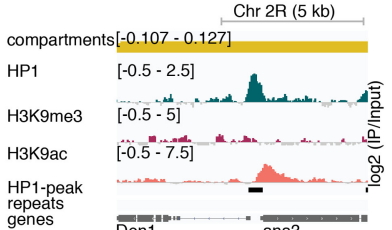

Extended Data Fig. 2 | Characterization of HP1 binding within A- and

B-compartment. a, Hi-C contact maps with contact frequencies of

chromosome $3 \mathrm{~L}(7-15 \mathrm{Mb})$ at a resolution of $40 \mathrm{~kb}$. Four out of seven biological

replicates are shown. $\mathbf{b}$, Representative example of HP1 binding in a

B-compartment region.c, Representative example of HP1 binding in an A-compartment region.d, Extended characterization of HP1 binding in A-compartment regions. The heat maps show ChIP-seq signal and repeat coordinates in $\pm 10 \mathrm{~kb}$ centred around HP1 peaks. Figure $2 \mathrm{~d}$ shows only cluster 2 containing HP1 peaks that localize within non-repetitive, active regulatory sequences enriched in $\mathrm{H} 3 \mathrm{~K} 9 \mathrm{ac}, \mathrm{H} 3 \mathrm{~K} 27 \mathrm{ac}, \mathrm{H} 3 \mathrm{~K} 4 \mathrm{mel} / 3$ as well as polymerase II.

We validated this cluster in active regions by performing ChIP-seq with different antibodies against HP1 (HA antibody against a Flag-HA-HP1-tagged transgene (second heat map) and HP1 Covance antibody (third heat map)). We further performed ChIP-seq in HP1-KD embryos using $\lambda$-DNA spike-in to normalize the signal and found a strong reduction of HP1 binding. This further validates the specificity of the HP1 peaks. See Supplementary Methods for further information. A second cluster of HP1 binding events (cluster 1 ) occurs in repetitive chromatin regions that are largely devoid of active histone modification marks. 


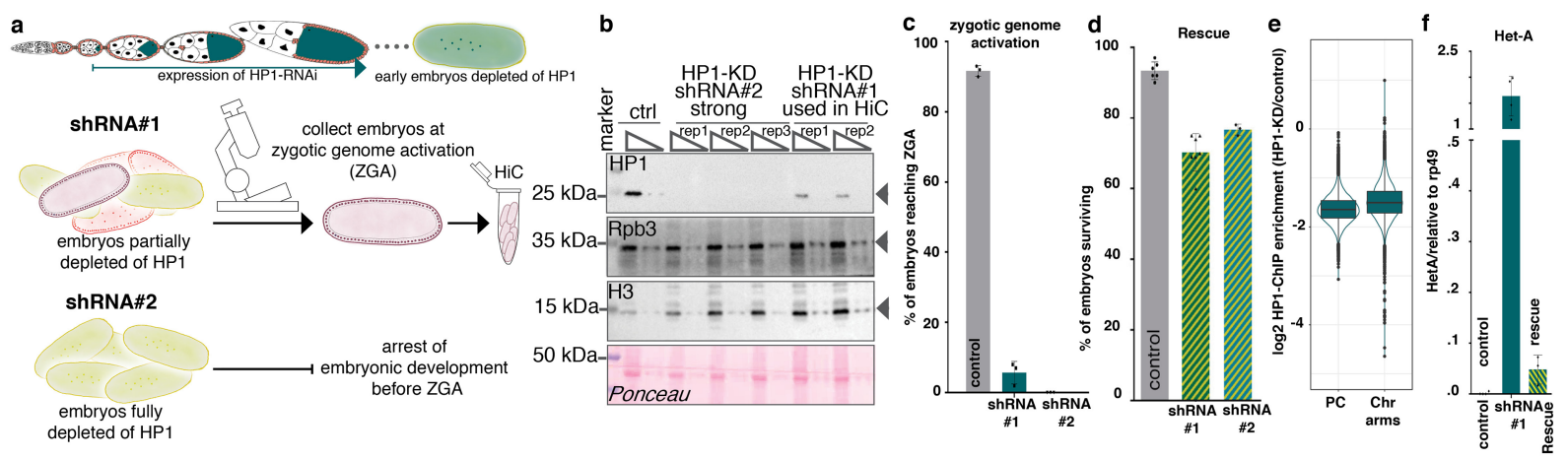

g control HP1-KD h chromosome 2R 6-25 Mb
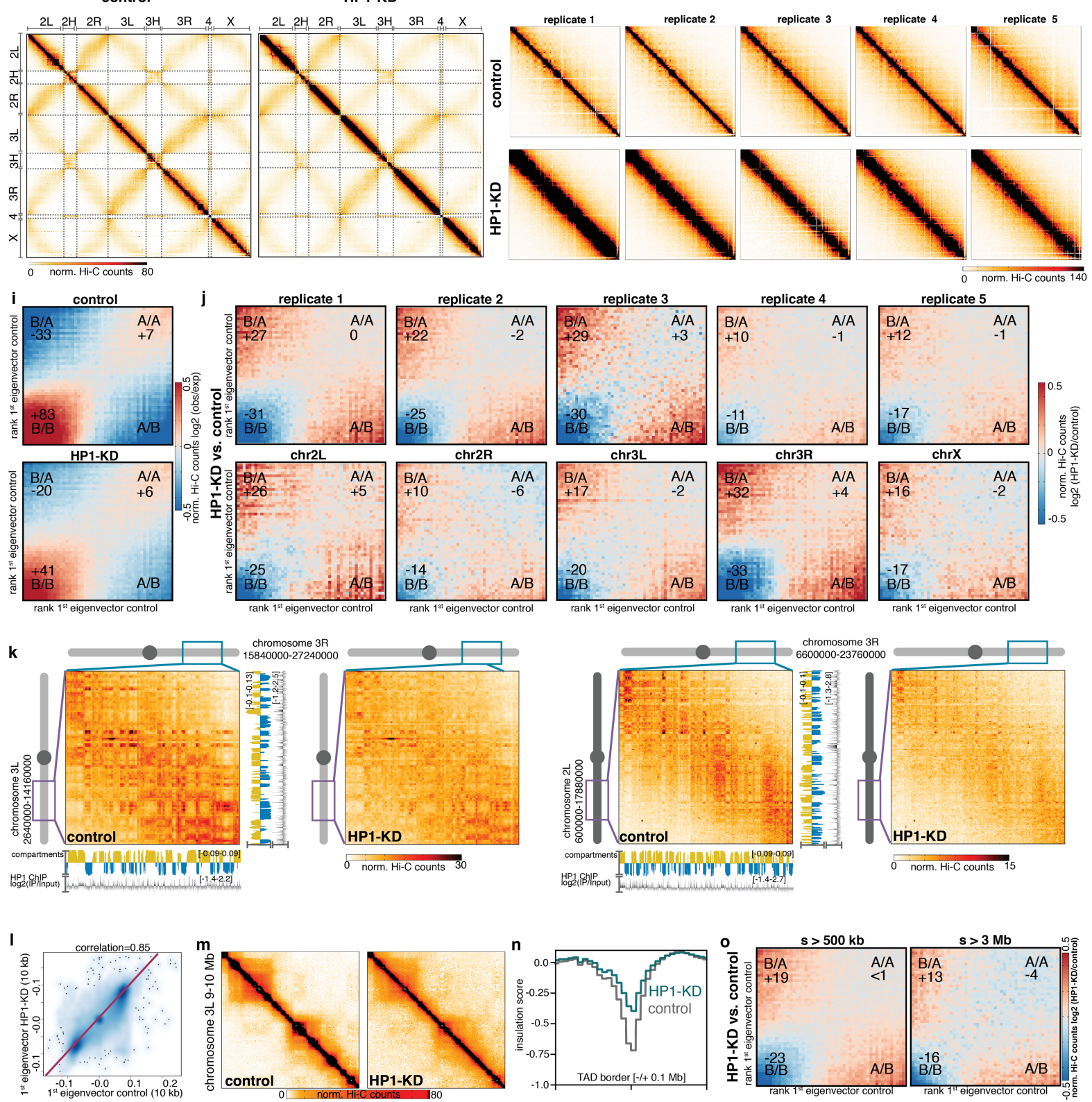

Extended Data Fig. 3 |See next page for caption. 
Extended Data Fig. 3 | Characterization of HP1 knockdown and its effect on 3D genome organization. a, Schematic of the mode of action of the RNA interference (RNAi) knockdown. shRNA against HP1 is expressed only at late stages of oogenesis and does not interfere with the production of fertilized embryos. The resulting early embryo is devoid of maternally loaded mRNA and protein. The bottom part shows the two knockdowns and embryo collection strategy. b. Western blot showing reduction of HP1 protein in early embryos after shRNA-mediated knockdown. shRNA\#1 was used to perform the Hi-C experiments and generated embryos carrying residual HP1; shRNA\#2 completely depleted $\mathrm{HP} 1$ proteins. $\mathrm{Rbp} 3, \mathrm{H} 3$ and Ponceau staining were used as loading controls. Representative of two independent experiments. For western blot source data, see Supplementary Fig. 1.c, Following the use of shRNA\#1, between $5 \%$ and $10 \%$ of the embryos reach ZGA, therefore allowing the study of 3D chromatin conformation. shRNA\#2 blocked embryonic development at the first or second mitotic division, with $0 \%$ embryos reaching ZGA, therefore preventing the study of the 3D chromatin conformation establishment. Data are mean \pm s.d. Number of biological replicates: 3 for control; 3 for HP1-KD shRNA\#1; 3 for HP1-KD shRNA\#2. d, Both shRNA\#1 and ShRNA\# 2 are specific towards HP1 depletion as both can be rescued by a FlagHA-tagged HP1-rescue construct (Extended Data Fig. 4a). Data are mean \pm s.d. Number of biological replicates: 6 for control; 7 for HP1-KD shRNA\#1; shRNA\#3 for HP1-KD shRNA\#2.e, Box plot showing reduction of the HP1ChIP-seq signal in HP1-KD embryos at zygotic genome activation on HP1 peaks at pericentromeric (PC) regions (left) and on HP1-peaks along the chromosome (Chr) arms (right). The signal is overall more reduced within pericentromeric regions compared to peaks along the chromosome arms. For comparison, quantitative ChIP-seq data using spike-in normalization have been used.See Supplementary Methods for definition of the pericentromeric regions. Box plots are as in Fig. 1d.f, Quantitative PCR (qPCR) measuring the upregulation of the telomeric repeat element Het-A caused by HP1-KD. The overexpression of Het-A can be rescued by the introduction of a HP1-rescue construct, that cannot be targeted by the hairpin. Data are mean \pm s.d. Number of biological replicates: $n=4$ for control; $n=4$ for HP1-KD; 3 for HP1-rescue. $g$, Genome-wide $\mathrm{Hi}-\mathrm{C}$ contact maps of control (left, 7 replicates) and HP1-KD (right, 5 replicates) embryos. h, Hi-C contact map in control (top) and HP1-KD (bottom) embryos across chromosome $2 \mathrm{R} 6-25 \mathrm{Mb}$ at a resolution of $120 \mathrm{~kb}$. Five biological replicates are shown. $\mathbf{i}$, Hi-C contact enrichment in control (top) and HP1-KD (bottom) embryos, sorted by compartment score showing strong decrease in B-compartment contacts and gain in in $\mathrm{A} / \mathrm{B}$ intermingling upon depletion of HP1. Quantification of the enrichment in compartment interactions is indicated in the respective corner of the plot. See Supplementary Methods for further details.j, Differential Hi-C contact enrichment in HP1-KD versus control $\mathrm{Hi}-\mathrm{C}$ maps, sorted by compartment score for all individual replicates used in the study (top) and the individual chromosome arms (bottom), confirming the consistency of the phenotype across replicates and chromosome arms. $\mathbf{k}, \mathrm{Hi}-\mathrm{C}$ contact maps in control (left) and HP1-KD (right) embryos showing the interarm interactions (3L 2640000-14160000 and 3R 15840000-27240000) of chromosome 3 (left) as well as inter-chromosome interactions between chromosome 2L (6000000-17880000) and chromosome 3R (660000023760000). In both cases, contacts and compartmentalization are strongly reduced after HP1 knockdown. I, Scatter plot of compartment scores (first eigenvector values at 10-kb resolution) in control and HP1-KD embryos (Spearman correlation 0.85), indicating the complete absence of compartment switches between control and HP1-KD embryos. m, Hi-C contact map across a 1 -Mb region on chr $3 \mathrm{~L}$, showing decreased insulation across topologically associating domains in HP1-KD embryos. $n$, Insulation scores in $\pm 100 \mathrm{~kb}$ surrounding TAD boundaries (Supplementary Methods) showing decreased insulation after HP1 depletion. o, Differential Hi-C contact enrichment in HP1$\mathrm{KD}$ versus control $\mathrm{Hi}-\mathrm{C}$ maps, sorted by compartment score for regions further apart than $500 \mathrm{~kb}$ (left) and regions further apart than $3 \mathrm{Mb}$ (right).

B-compartment contacts are also decreased at distances that exceed typical TAD sizes in Drosophila, which confirms that the moderately decreased insulation cannot account for the loss of B-compartment interactions. 

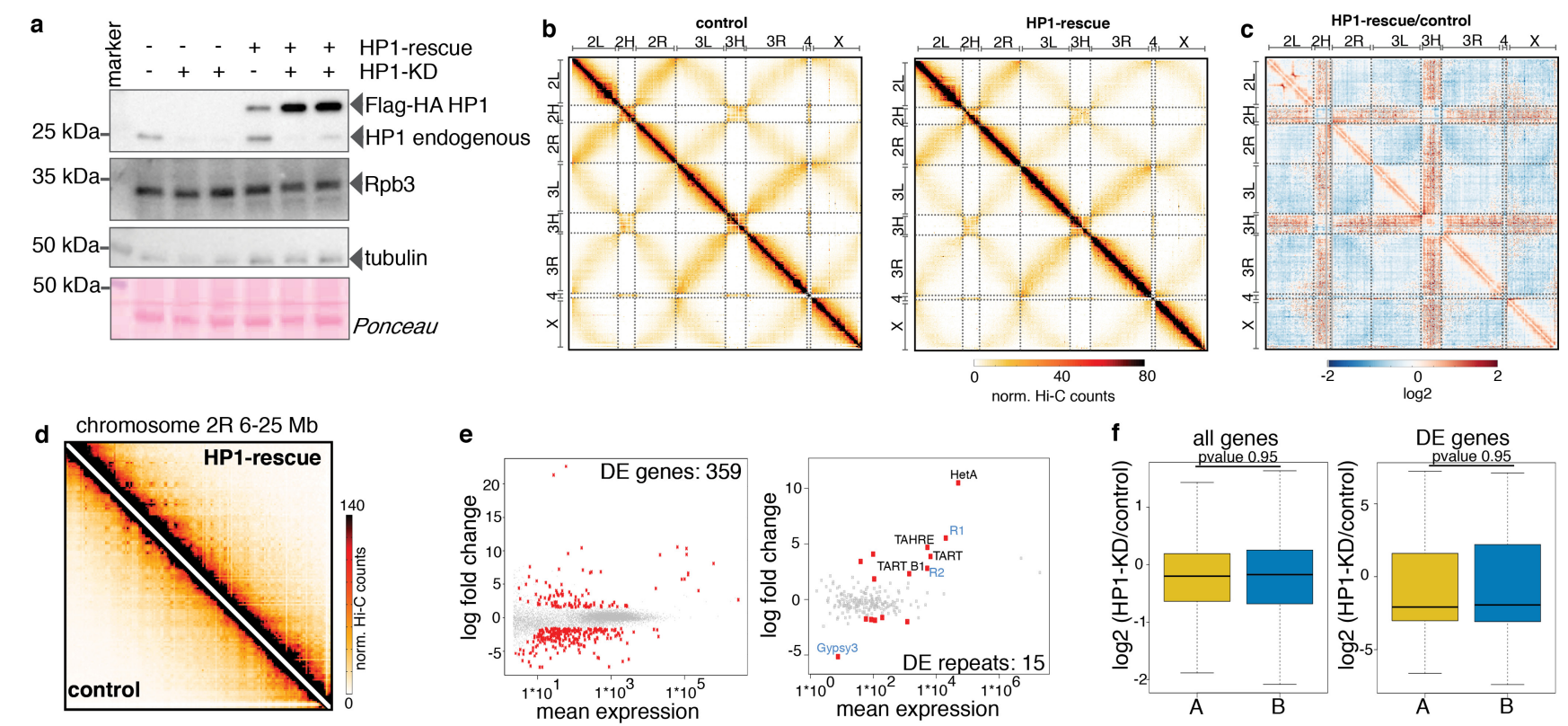

g
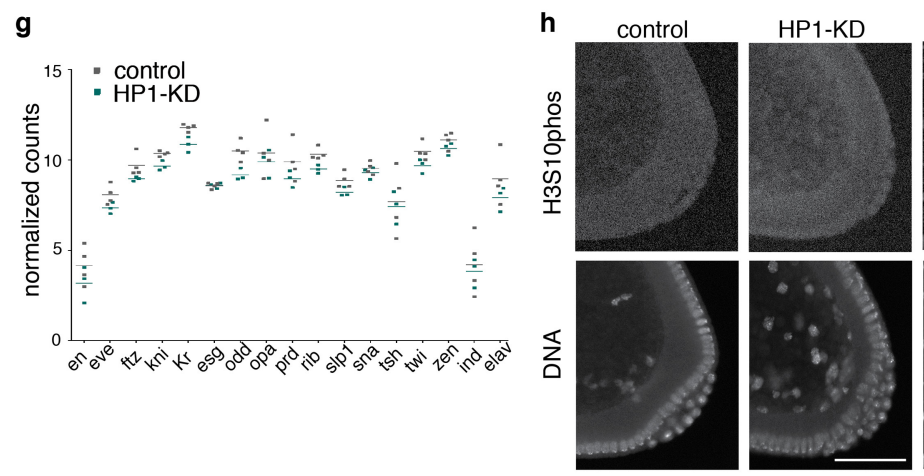

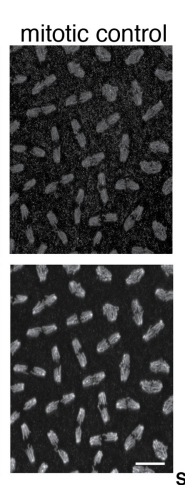

m

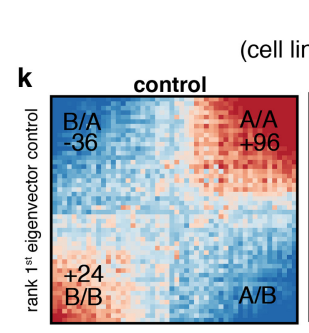

S2 cells
(cell line derived from late embryos)
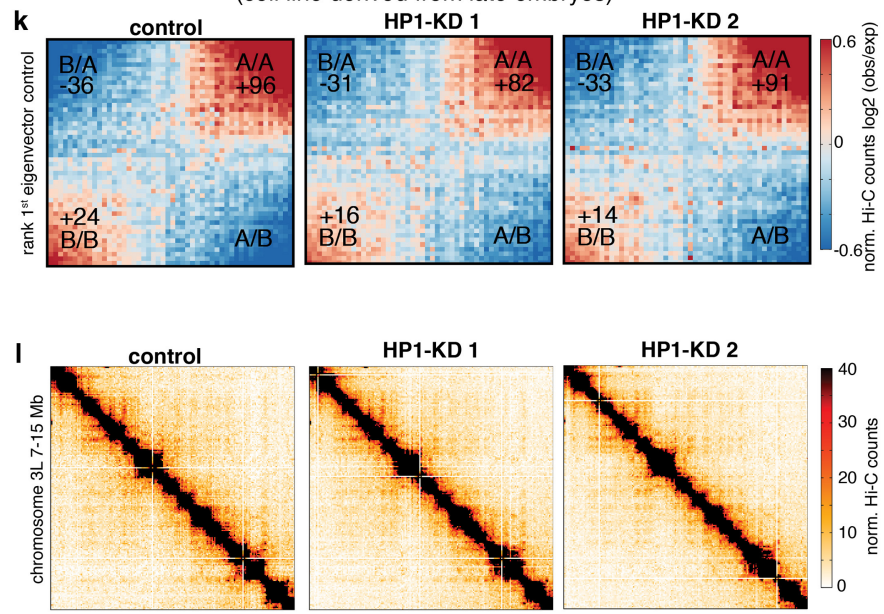

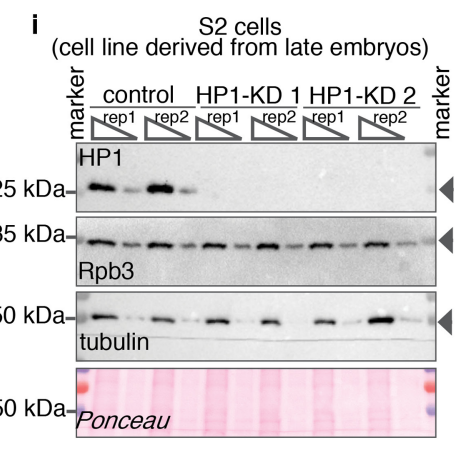

j

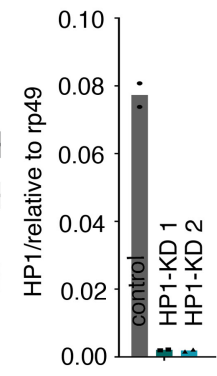

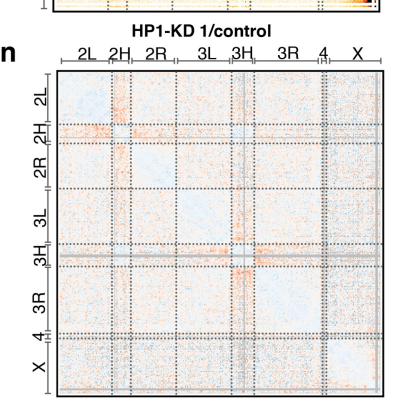
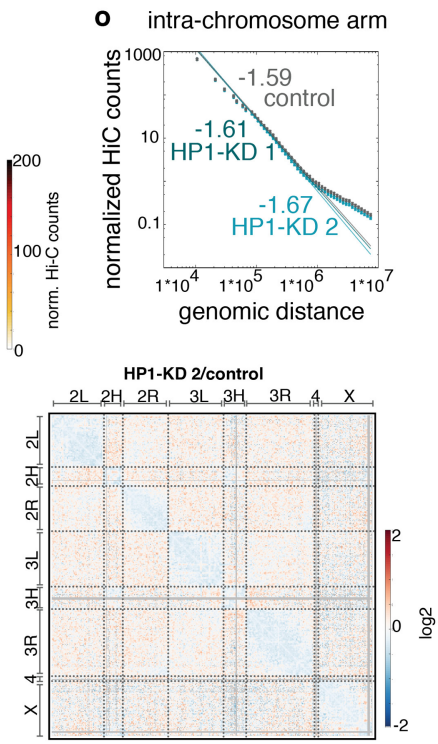

Extended Data Fig. 4 | See next page for caption. 
Extended Data Fig. 4 | Characterization of HP1 rescue, transcriptomic changes after HP1 knockdown and its effect on 3D genome organization in differentiated S2 cells. a, Western blot showing the expression of the Flag-HAtagged HP1 transgene in the background of control and HP1-KD embryos. Rpd3, tubulin and Ponceau were used as loading controls. After depletion of endogenous HP1, the expression of the transgene is increased. Blots are representative of two independent experiments. For western blot source data, see Supplementary Fig. 1.b, Genome-wide Hi-C contact maps in control (left) and HP1-rescue (right) embryos (40-kb resolution). The HP1-rescue and HP1-KD embryos show an inversion on chromosome 2L.c, Genome-wide differential $\mathrm{Hi}$ - $\mathrm{C}$ contact maps ( $\log _{2}$-transformed fold change) in HP1-rescue versus control embryos. The HP1-rescue construct reverses the structural effects of HP1-KD (reduced contact frequency between the pericentromeric regions, as well as inter-chromosome arm interactions and compaction defects). d, Same genomic region as in Fig. $3 \mathrm{c}$, d, with control and HP1-KD embryos expressing HP1-rescue.e, Left, MA plot illustrating differential expression of genes at zygotic genome activation in HP1-KD versus control embryos. In total, we detected 359 differentially expressed genes using RNA-seq (red dots) (Supplementary Methods) (of the total 277 genes are in A-compartment, 72 genes are in the B-compartment regions and 10 genes are on chrUn CP007120v1). Right, MA plot showing the differential expression of types of repeat. We detected 15 differentially expressed repeat types, highlighted in the plot (Supplementary Methods).f, Box plot showing the distribution of gene expression changes within A-and B-compartments. We did not detect any differences in the distribution of gene expression changes in $\mathrm{A}$ - and B-compartments either considering all genes (left, $P=0.95$, one-sided Wilcoxon test) or only significant differentially expressed genes (right, $P=0.95$, one-sided Wilcoxon test). Box plots are as in Fig. 1d; outliers not shown.

g, Expression of a panel of 17 purely zygotically expressed transcription factors in control and HP1-knockdown embryos. In unfertilized eggs all factors are not expressed and become upregulated at zygotic genome activation. The expression of the zygotic transcription factors confirms that HP1-KD embryos undergo zygotic genome activation. Each dot represents the normalized counts for a given transcription factor of a replicate RNA sequencing (RNA-seq) experiment. h, Immunofluorescence staining of control and HP1-KD embryos at zygotic genome activation with the mitosis marker $\mathrm{H} 3 \mathrm{~S} 10$ phosphorylated.
Until the cellular blastoderm stage (ZGA), all nuclei undergo mitosis synchronously and then enter $\mathrm{G} 2$ phase at ZGA. The ratio of mitotic cells and the timing of mitosis is not altered in HP1-KD embryos. Scale bar, $50 \mu \mathrm{m}$. As a control for antibody specificity, an earlier stage of embryogenesis (before ZGA) was stained showing a strong H3S10phospho signal after synchronous entry into mitosis (right). Representative images from three biological replicates. Scale bar, $10 \mu \mathrm{m}$. i, Western blot showing the reduction of HP1 after treatment with double-stranded RNA (dsRNA) treatment in S2 cells (cell culture cells derived from a primary culture of late-stage (20-24 h old) Drosophila embryos, probably from a macrophage-like lineage). Rpd3, tubulin and Ponceau were used as loading controls. To control for unspecific effects of the dsRNA treatment, control cells were treated with a dsRNA against glutathione $S$-transferases (GST) and two different dsRNAs were used to deplete HP1. Representative of two independent experiments. For western blot source data, see Supplementary Fig. 1. See Supplementary Methods for further details. j, qPCR analysis showing the reduction of HP1 mRNA after dsRNA treatment in S2 cells. The signal is relative to $r p 49$. To control for unspecific effects of the dsRNA treatment, control cells were treated with a dsRNA against GST and two different dsRNAs were used to deplete HP1. See Supplementary Methods for further details. Data are mean of two independent experiments. $\mathbf{k}$, Hi-C contact enrichment in control (left) and HP1-KD (right) in S2 cells, sorted by compartment score, showing no decrease in B-compartment contacts after depletion of HP1 with either dsRNA. This indicates that HP1 is required for the establishment of the B-compartment during early embryonic development but does not affect the maintenance of compartmentalization in late differentiated cells. $\mathrm{I}, \mathrm{Hi}-\mathrm{C}$ contact frequencies of a $19-\mathrm{Mb}$ region on chromosome $3 \mathrm{~L}$ at a resolution of $120 \mathrm{~kb}$. Pooled $\mathrm{Hi}-\mathrm{C}$ data of two biological replicates are shown. $\mathbf{m}$, Genome-wide Hi-C contact map in control S2 cells ( $120-\mathrm{kb}$ resolution). n, Genome wide differential $\mathrm{Hi}$ - $\mathrm{C}$ contact maps ( $\log _{2}$-transformed fold change) in HP1-KD versus control S2 cells. The differential contact maps show the HP1KD with two independent shRNA on the left and right, respectively. o, Contact probabilities over the genomic distance of control and HP1-KD S2 cells. The contact probability of the HP1-KD cells closely resembles the control. Pericentromeric regions were excluded from the analysis of contact probabilities (Supplementary Methods). 


\section{Article}
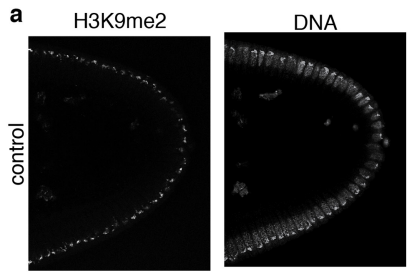

b pericentromeric chromosome

HP1 ChIP signal spike in normalized

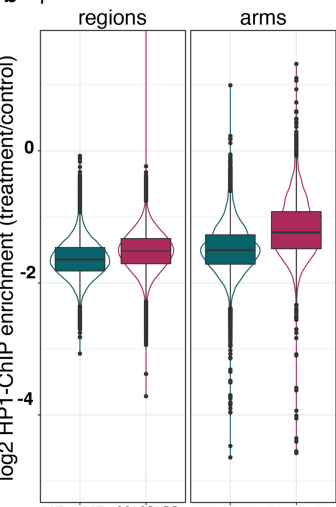

e HP1 HP1-CD H3K9me3

HP1-KD H3K9M HP1-KD H3K9M
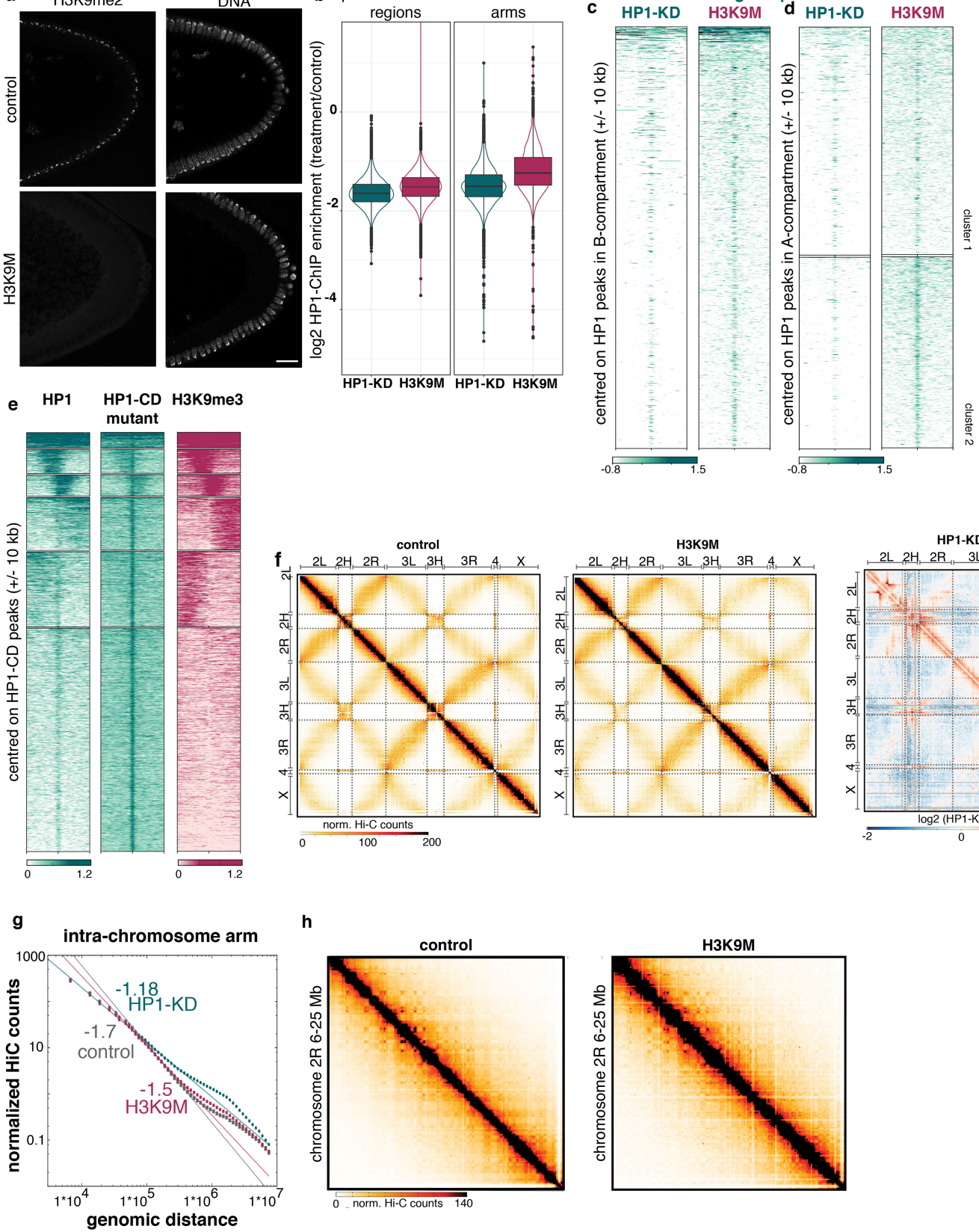

h
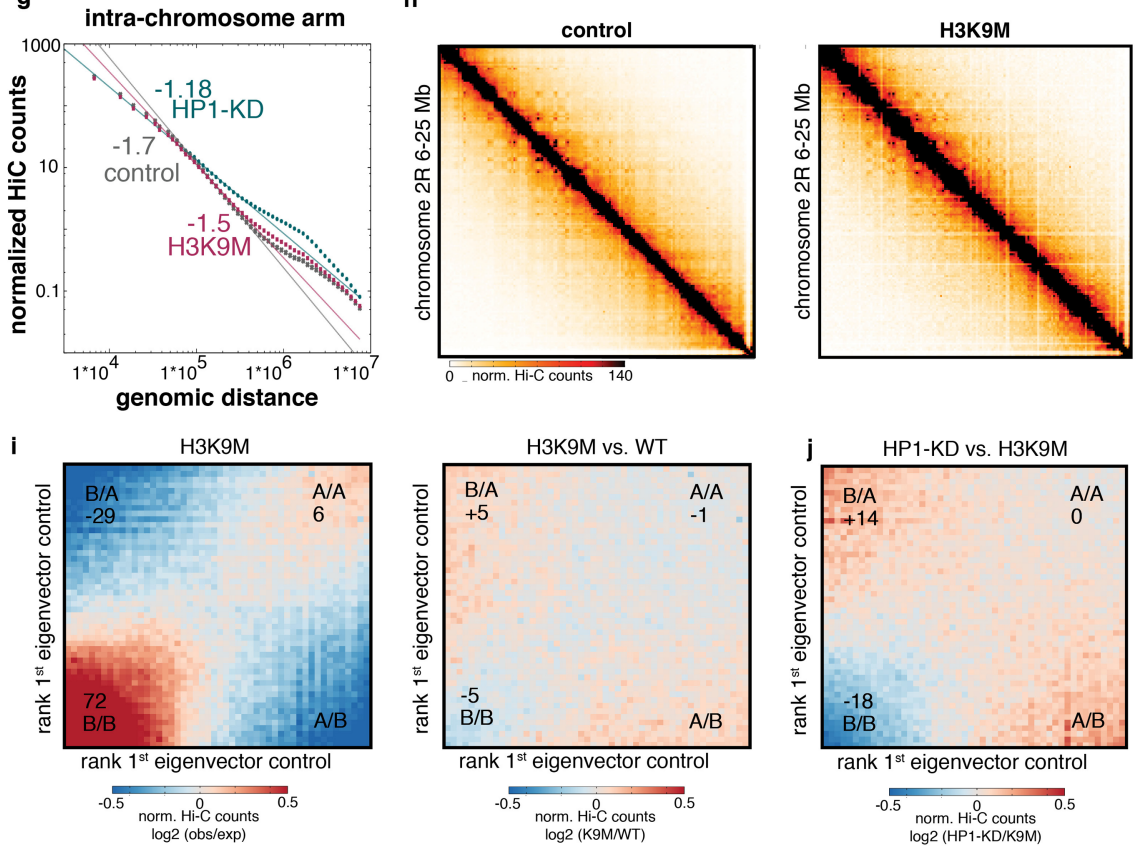

Extended Data Fig. 5 | See next page for caption. 
Extended Data Fig. 5 | Characterization of H3K9M. a, Immunofluorescence staining of embryos at ZGA showing that $\mathrm{H} 3 \mathrm{~K} 9 \mathrm{me} 2$ is completely lost after expression of the H3K9M mutant. H3K9M depletes H3K9me2/3 from chromatin, and acts as a competitive inhibitor of the histone methyltransferases. Representative image from three biological replicates. Scale bar, $20 \mu \mathrm{m}$. b, Box plot showing the reduction of the HP1 ChIP-seq signal over the control embryos at ZGA at HP1 peaks in HP1-KD (green) and H3K9M (red) embryos. The signal is reduced overall in HP1-KD embryos, with more loss in the pericentromeric region (left) compared to chromosome arms (right). For comparison, quantitative ChIP-seq data using spike-in normalization has been used. Box plots are as in Fig. 1d. c, Characterization of HP1 binding in B-compartment regions after HP1 knockdown (left) and H3K9M overexpression (right). Heat maps of HP1ChIP-seq signals are $\pm 10 \mathrm{~kb}$ centred on HP1 peaks and show that HP1 is retained at a higher level in $\mathrm{H} 3 \mathrm{~K} 9 \mathrm{M}$ embryos compared to the HP1-KD embryos. Spike-in normalization has been used to quantify the enrichment. d, As in c, but in A-compartment regions. e, Characterization of HP1 binding in ovaries. Left, binding of a control HP1-Flag-HA-tagged transgene. Middle, binding of a Flag-HA-tagged chromodomain mutant of HP1(HP1-CD) that cannot bind to H3K9me2/3. Right, the enrichment of $\mathrm{H} 3 \mathrm{~K} 9 \mathrm{me} 3$ in ovaries. The heat maps of HP1 ChIP-seq signals are $\pm 10 \mathrm{~kb}$ centred on HP1 peaks called in the HP1 chromodomain mutant. f, Genome-wide Hi-C contact maps in control (left) and H3K9M (middle) embryos (120-kb resolution, pooled $\mathrm{Hi}-\mathrm{C}$ data of two biological replicates). Right, differential $\mathrm{Hi}$-C contact map ( $\log _{2}$-transformed fold change in HP1-KD versus $\mathrm{H} 3 \mathrm{~K} 9 \mathrm{M}$ ), highlighting milder compaction within arms in $\mathrm{H} 3 \mathrm{~K} 9 \mathrm{M}$ with respect to HP1-KD. g, H3K9M shows decay of contact probability similar to control embryos within arms. This suggests that compaction in the $\mathrm{H} 3 \mathrm{~K} 9 \mathrm{M}$ mutant is milder than in HP1-KD embryos. $\mathbf{h}, \mathrm{Hi}-\mathrm{C}$ contact maps on chr2R (6-25 Mb) in control and $\mathrm{H} 3 \mathrm{~K} 9 \mathrm{M}$ embryos (120-kb resolution). $\mathbf{i}, \mathrm{Hi}-\mathrm{C}$ contact enrichment in $\mathrm{H} 3 \mathrm{~K} 9 \mathrm{M}$ (left) and differential Hi-C contact enrichment in $\mathrm{H} 3 \mathrm{~K} 9 \mathrm{M}$ versus control (right) in embryos, sorted by compartment score showing no decrease in B-compartment contacts upon H3K9M expression.j, Differential $\mathrm{Hi}-\mathrm{C}$ contact enrichment in $\mathrm{HP1}-\mathrm{KD}$ versus $\mathrm{H} 3 \mathrm{~K} 9 \mathrm{M} \mathrm{Hi}-\mathrm{C}$ maps, sorted by compartment score. B-compartment interactions are more strongly decreased in HP1-KD embryos than in H3K9M embryos. 
a Control Mutant

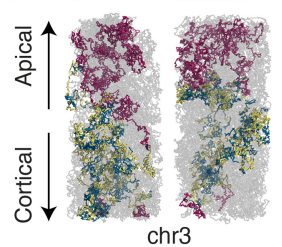

chr3
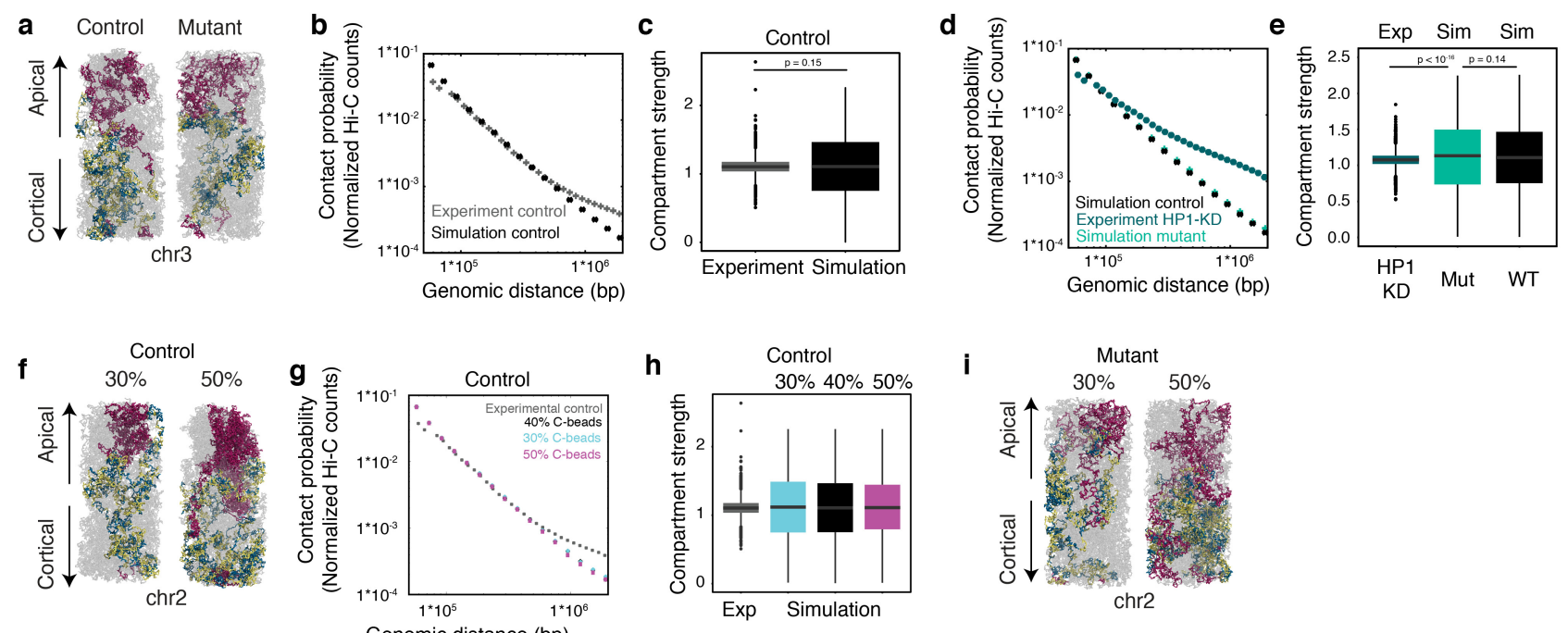

Genomic distance (bp)
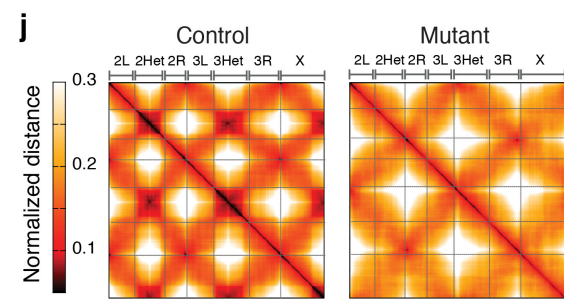

Mutant/Control

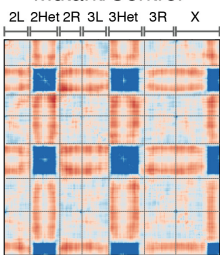

$30 \%$ C-beads
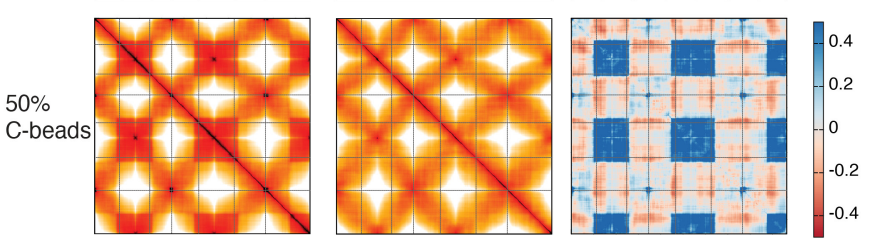

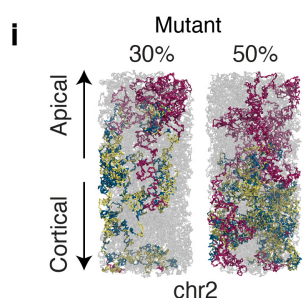

k

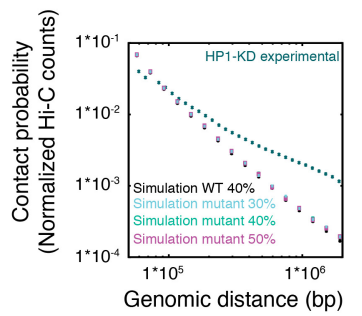

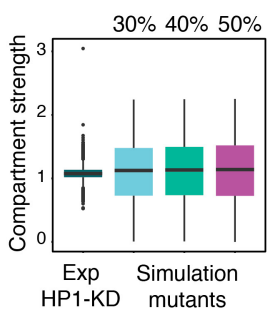

Extended Data Fig. 6 | Genome-wide simulations show that loss of HP1 at pericentromeric regions does not cause the phenotype within chromosome arms. a, Snapshots of control (left) and mutant (decreased interactions between C-type beads and between C-type beads and the nuclear envelope, right) simulations, reproducing the experimental scaling and compartment strength. Colour code as in Fig. 4 a. b, Scaling of contact probabilities in experimental and simulated contact maps.c, Simulated and experimental compartment strength. Pvalues determined by two-sided Wilcoxon test.d, Scaling of contact probabilities in experimental HP1-KD and simulated control embryos and mutant contact maps. No differences between simulated control and mutant are detected.e, Experimental compartment strength in HP1-KD and simulated compartment strength in control and mutant samples. No differences between simulated control and mutant are detected. $P$ values determined by two-sided Wilcoxon test.f, Snapshots of the control simulations with different amounts of C-type beads (30\% and 50\%). A single full chromosome is highlighted in each snapshot. g, Scaling of contact probabilities in experimental and simulated contact maps with different amounts of C-type beads. $\mathbf{h}$, Experimental and simulated compartment strength with different amounts of C-type beads. i, Snapshots of the simulations with decreased interactions between C-type beads and their interaction with nuclear surface. A single chromosome is highlighted in each snapshot. Different amounts of C-type beads (30\% and $50 \%$ of the total number of beads) are shown.j, Genome-wide simulated distance maps of control (left) and mutant samples with decreased interaction within and between C-type beads and their interaction with nuclear surface (middle). Differential distance map ( $\log _{2}$-transformed fold change in mutant versus control) highlighting increased distance within and between centromeric and telomeric regions (right). Different amounts of C-type beads (30\% and $50 \%$ of the total number of beads) are shown. $\mathbf{k}$, Scaling of contact probabilities in experimental HP1-KD and simulated control and mutant contact maps with different amounts of C-type beads. No differences between simulated control and mutants are detected. I, Experimental compartment strength in HP1-KD and simulated compartment strength in mutants with different amounts of C-type beads. No differences between the simulated mutants are detected. Box plots are as in Fig.1d. 

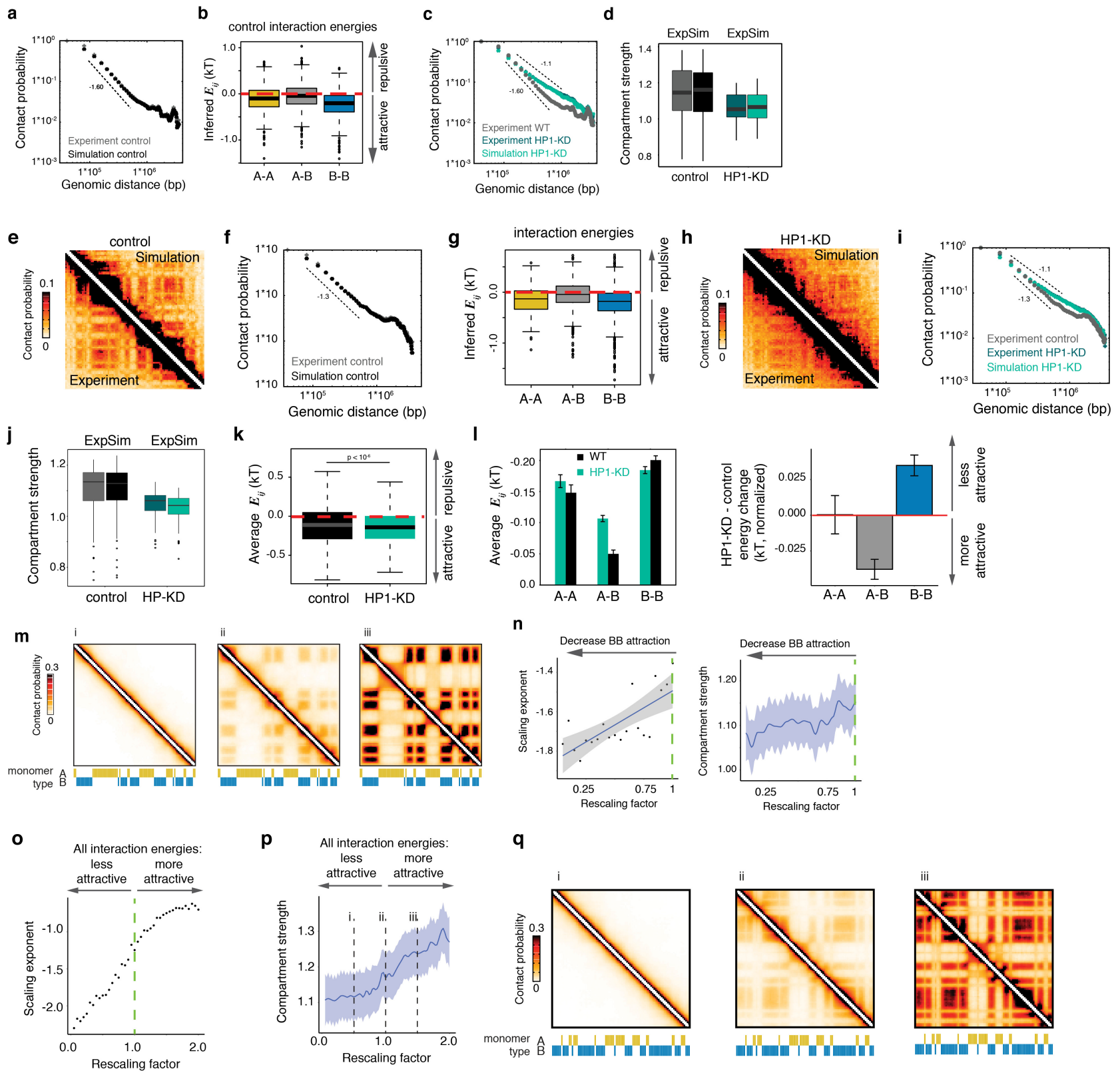

9

$r$
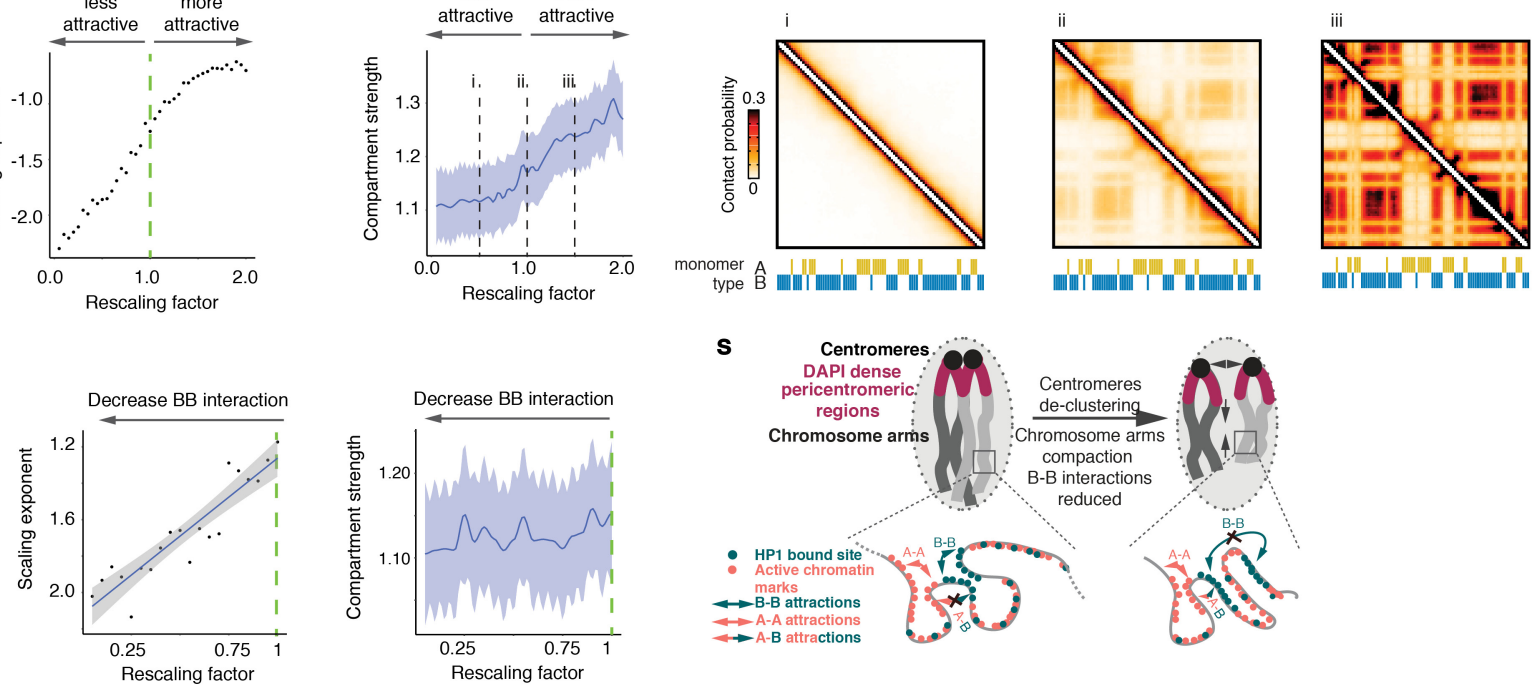

S
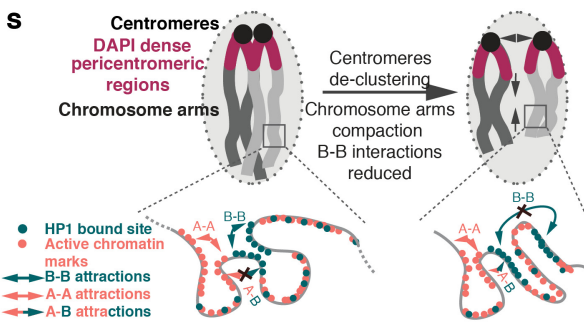

Extended Data Fig. 7 |See next page for caption. 


\section{Article}

Extended Data Fig. 7 |HP1-KD phenotype is driven by two independent mechanisms, both mediated by HP1. a, Scaling of contact probabilities in experimental and simulated contact maps shown in Fig. 4e. b, Inferred interaction energies between pairs of beads are overall attractive, with interactions between $B$-compartment beads being more attractive than interactions between A-A and A-B beads. Simulated region: chr3R 17-20.6 Mb. c, Scaling of contact probabilities in experimental and simulated $\mathrm{Hi}-\mathrm{C}$ maps, compared to the scaling in the experimental control $\mathrm{Hi}-\mathrm{C}$ heat map as a reference. Simulated region: chr3R 17-20.6 Mb. d, Simulated and experimental compartment strength (chr3R 17-20.6 Mb).e, Experimental and simulated contact maps of control embryos. Simulated region: chr3R 25.4-29 Mb.

f, Scaling of contact probabilities in experimental and simulated contact maps shown in $\mathbf{e}$. g, Inferred interaction energies between pairs of beads are overall attractive, with at tractions between $B$-compartment beads being more attractive than interactions between $\mathrm{A}-\mathrm{A}$ and $\mathrm{A}-\mathrm{B}$ beads. Simulated region: chr3R 25.4-29 Mb. h, Experimental and simulated HP1-KD contact maps in the same region as in $\mathbf{e} . \mathbf{i}$, Scaling of contact probabilities in experimental and simulated $\mathrm{Hi}-\mathrm{C}$ maps, compared to the scaling in the experimental control. Simulated region: chr3R 25.4-29 Mb.j, Simulated and experimental compartment strength in the same chr3R region. Simulated region: chr3R 25.4-29 Mb. k, Inferred interaction energies are overall more attractive in the HP1-KD model. $P$ value determined by two-sided Wilcoxon test. Simulated region: chr3R 25.4-29 Mb. I, Left, interaction energies between B-compartment type beads become comparatively less attractive, whereas interactions between A-type compartment beads and between A- and B-type beads become more attractive. Data are mean and s.e.m. across each interaction energy class (number of interactions: 465 for A-A; 1,858 for A-B; and 1,769 for $B-B$ ). Right, average changes in inferred interaction energies between HP1-KD and the control models, classified according to whether they are within or across A- and B-compartment regions. Attractions between B-compartment regions are decreased in the HP1-KD model. Data are mean and s.e.m. across each interaction energy class (number of interactions: 465 for
$\mathrm{A}-\mathrm{A} ; 1,858$ for $\mathrm{A}-\mathrm{B}$; and 1,769 for $\mathrm{B}-\mathrm{B})$. Average changes in inferred interaction energies between HP1-KD and the control models, irrespective of being within or across A- and B-compartment regions, are set to zero. Interaction energies between $B$-compartment type beads become less attractive, whereas energies between A-type compartment beads and between A and B become more attractive. $\mathbf{m}$, Example of the simulated contact maps for different levels of compartment strength, corresponding to different energy rescaling factors ( $i$, ii and iii, as in Fig. 4j). Arrangement of A and B beads based on: chr3R 17-20.6 Mb. $\mathbf{n}$, Same plot as in Fig. 4i, j, when only attractions between B-compartment regions are decreased. Mean (bold line) with the confidence interval (shaded area) calculated using t-based approximation is shown. Arrangement of $A$ and $B$ beads based on: chr3R 17-20.6 Mb. o, Scaling exponents in simulated contact maps are plotted against increasing or decreasing (by a multiplicative scaling factor) $\mathrm{A}-\mathrm{A}, \mathrm{A}-\mathrm{B}$ and $\mathrm{B}-\mathrm{B}$ attractive interaction. The scaling exponent increases when increasing attractions between all beads and vice versa. Arrangement of A and B beads based on chr3R 25.4-29 Mb. p, Compartment strength in simulated contact maps decreases upon increase in attractions between all types of bead, and vice versa. Mean (bold line) with the confidence interval (shaded area) calculated using t-based approximation is shown. Arrangement of A and B beads based on chr3R 25.4-29 Mb.q, Example of the simulated contact maps for different levels of compartment strength, corresponding to different energy rescaling factors. Arrangement of A and B beads based on chr3R 25.4-29 Mb. r, As in $\mathbf{o}$ and $\mathbf{p}$, when only attractions between B-compartment regions are decreased. Mean (bold line) with the confidence interval (shaded area) calculated using t-based approximation is shown. Arrangement of A and B beads based on chr3R 25.4-29 Mb. s, Proposed model in which chromatin-bound HP1 mediates B-B attractions. A-A attractions independent of HP1 promote establishment of the A-compartment. Depletion of HP1 causes pericentromeric region declustering and increased chromosome arm compaction. B-B interactions are reduced, leading to an overall increase in $\mathrm{A}-\mathrm{A}$ and $\mathrm{A}-\mathrm{B}$ attractive energies (Supplementary Methods, Extended Data Fig. 6). Box plots are as in Fig. 1d; outliers not shown in $\mathbf{k}$. 


\section{Reporting Summary}

Nature Research wishes to improve the reproducibility of the work that we publish. This form provides structure for consistency and transparency in reporting. For further information on Nature Research policies, see our Editorial Policies and the Editorial Policy Checklist.

\section{Statistics}

For all statistical analyses, confirm that the following items are present in the figure legend, table legend, main text, or Methods section.

n/a Confirmed

$\square$ The exact sample size $(n)$ for each experimental group/condition, given as a discrete number and unit of measurement

$\square$ A statement on whether measurements were taken from distinct samples or whether the same sample was measured repeatedly

$\square$ The statistical test(s) used AND whether they are one- or two-sided

$\square$ Only common tests should be described solely by name; describe more complex techniques in the Methods section.

$\square$ \ A description of all covariates tested

$\square$ \A description of any assumptions or corrections, such as tests of normality and adjustment for multiple comparisons

$\square$ A full description of the statistical parameters including central tendency (e.g. means) or other basic estimates (e.g. regression coefficient)

$\bigotimes$ AND variation (e.g. standard deviation) or associated estimates of uncertainty (e.g. confidence intervals)

$\varnothing$ For null hypothesis testing, the test statistic (e.g. $F, t, r$ ) with confidence intervals, effect sizes, degrees of freedom and $P$ value noted

Give P values as exact values whenever suitable.

$\square$ \ For Bayesian analysis, information on the choice of priors and Markov chain Monte Carlo settings

Х $\square$ For hierarchical and complex designs, identification of the appropriate level for tests and full reporting of outcomes

Х $\square$ Estimates of effect sizes (e.g. Cohen's $d$, Pearson's $r$ ), indicating how they were calculated

Our web collection on statistics for biologists contains articles on many of the points above.

\section{Software and code}

Policy information about availability of computer code

Data collection Western Blots were imaged using ChemiDoc Imaging System with Image-Lab(Bio-Rad), qPCR data was collected using LightCycler 96 (Roche) For immunofluorescence Zen (Zeiss) was used to record the data.

Data analysis QuasR v1.30, deepTools v2.4.2, MonteGrappa v1.2, HiC-Pro v2.11.1, HiTC v1.32.0, IDR v.2.0, reshape2 v1.4.4, rtracklayer v1.50.0, Rcpp v1.0.6, GenomicRanges v1.42.0, matrix2insulation.pl v1.0.0, MACS2 2.1.3.3, STAR v2.7.0, FIJI v.2.1.0, LAMMPS, GraphPad Prism 6, Imaris 9.5.1

(Bitplane) Further settings of the Hi-C, compartment analysis and the simulation have been made available here: https://github.com/ zhanyinx/Zenk_Zhan_et_al_Nature2021

For manuscripts utilizing custom algorithms or software that are central to the research but not yet described in published literature, software must be made available to editors and reviewers. We strongly encourage code deposition in a community repository (e.g. GitHub). See the Nature Research guidelines for submitting code \& software for further information.

\section{Data}

Policy information about availability of data

All manuscripts must include a data availability statement. This statement should provide the following information, where applicable:

- Accession codes, unique identifiers, or web links for publicly available datasets

- A list of figures that have associated raw data

- A description of any restrictions on data availability

All HiC, ChIP and RNA-Seq raw files generated in this study has been uploaded to GEO (GSE140542).

https://www.ncbi.nlm.nih.gov/geo/query/acc.cgi?acc=GSE140542

Also processed data are available there. The bed files of called HP1 peaks is provided as Supplementary Tables of this study. Further a table with RNA-Seq counts is 


\section{Field-specific reporting}

Please select the one below that is the best fit for your research. If you are not sure, read the appropriate sections before making your selection. $\bigotimes$ Life sciences Behavioural \& social sciences Ecological, evolutionary \& environmental sciences

For a reference copy of the document with all sections, see nature.com/documents/nr-reporting-summary-flat.pdf

\section{Life sciences study design}

All studies must disclose on these points even when the disclosure is negative.

Sample size

For Hi-C data we used 5-7 biological replicates. All ChIP-Seq experiments have been performed at least in biological replicates. For RNA-Seq 3-4 biological replicates were collected. We did not apply statistical methods to pre-determine sample size and followed the general standard practice in the field. Number of replicate experiments is indicated in the legends.

Data exclusions We did not exclude data.

Replication We performed all experiments in biological replicates and could observe agreement between the replicates. All experiments were performed at least twice independently and material was collected independently and by different researchers.

Randomization We controlled variability by collecting the samples in several batches and by employing different researchers. Samples were allocated randomly to the researcher. We also performed a high number of biological replicates and collected pools of embryos at the same developmental stage.

Blinding We did not perform blinded experiments. Complete blinding was not possible because the mutant phenotypes were evident from the development of the embryo. First computational analysis and inspection of $\mathrm{HiC}$ data were performed blinded.

\section{Behavioural \& social sciences study design}

All studies must disclose on these points even when the disclosure is negative.

Study description

Research sample

Sampling strategy

Data collection

Timing

Data exclusions

Non-participation

Randomization
Briefly describe the study type including whether data are quantitative, qualitative, or mixed-methods (e.g. qualitative cross-sectional, quantitative experimental, mixed-methods case study).

State the research sample (e.g. Harvard university undergraduates, villagers in rural India) and provide relevant demographic information (e.g. age, sex) and indicate whether the sample is representative. Provide a rationale for the study sample chosen. For studies involving existing datasets, please describe the dataset and source.

Describe the sampling procedure (e.g. random, snowball, stratified, convenience). Describe the statistical methods that were used to predetermine sample size OR if no sample-size calculation was performed, describe how sample sizes were chosen and provide a rationale for why these sample sizes are sufficient. For qualitative data, please indicate whether data saturation was considered, and what criteria were used to decide that no further sampling was needed.

Provide details about the data collection procedure, including the instruments or devices used to record the data (e.g. pen and paper, computer, eye tracker, video or audio equipment) whether anyone was present besides the participant(s) and the researcher, and whether the researcher was blind to experimental condition and/or the study hypothesis during data collection.

Indicate the start and stop dates of data collection. If there is a gap between collection periods, state the dates for each sample cohort.

If no data were excluded from the analyses, state so OR if data were excluded, provide the exact number of exclusions and the rationale behind them, indicating whether exclusion criteria were pre-established.

State how many participants dropped out/declined participation and the reason(s) given OR provide response rate OR state that no participants dropped out/declined participation.

If participants were not allocated into experimental groups, state so OR describe how participants were allocated to groups, and if allocation was not random, describe how covariates were controlled. 


\section{Ecological, evolutionary \& environmental sciences study design}

All studies must disclose on these points even when the disclosure is negative.
Study description
Briefly describe the study. For quantitative data include treatment factors and interactions, design structure (e.g. factorial, nested, hierarchical), nature and number of experimental units and replicates.

Research sample

\section{Describe the research sample (e.g. a group of tagged Passer domesticus, all Stenocereus thurberi within Organ Pipe Cactus National} Monument), and provide a rationale for the sample choice. When relevant, describe the organism taxa, source, sex, age range and any manipulations. State what population the sample is meant to represent when applicable. For studies involving existing datasets, describe the data and its source.

Sampling strategy

Note the sampling procedure. Describe the statistical methods that were used to predetermine sample size OR if no sample-size calculation was performed, describe how sample sizes were chosen and provide a rationale for why these sample sizes are sufficient.

Data collection

Describe the data collection procedure, including who recorded the data and how.

Timing and spatial scale

Indicate the start and stop dates of data collection, noting the frequency and periodicity of sampling and providing a rationale for these choices. If there is a gap between collection periods, state the dates for each sample cohort. Specify the spatial scale from which the data are taken

Data exclusions

If no data were excluded from the analyses, state so OR if data were excluded, describe the exclusions and the rationale behind them, indicating whether exclusion criteria were pre-established.

Reproducibility

Describe the measures taken to verify the reproducibility of experimental findings. For each experiment, note whether any attempts to repeat the experiment failed $O R$ state that all attempts to repeat the experiment were successful.

Randomization

Describe how samples/organisms/participants were allocated into groups. If allocation was not random, describe how covariates were controlled. If this is not relevant to your study, explain why.

Blinding

Describe the extent of blinding used during data acquisition and analysis. If blinding was not possible, describe why OR explain why blinding was not relevant to your study.

Did the study involve field work? $\square$ Yes $\square$ No

\section{Field work, collection and transport}

Field conditions

Describe the study conditions for field work, providing relevant parameters (e.g. temperature, rainfall).

Location

State the location of the sampling or experiment, providing relevant parameters (e.g. latitude and longitude, elevation, water depth).

Access \& import/export

Describe the efforts you have made to access habitats and to collect and import/export your samples in a responsible manner and in compliance with local, national and international laws, noting any permits that were obtained (give the name of the issuing authority, the date of issue, and any identifying information).

Disturbance

\section{Reporting for specific materials, systems and methods}

We require information from authors about some types of materials, experimental systems and methods used in many studies. Here, indicate whether each material, system or method listed is relevant to your study. If you are not sure if a list item applies to your research, read the appropriate section before selecting a response.

Materials \& experimental systems

\begin{tabular}{|c|c|}
\hline$n / a$ & Involved in the study \\
\hline & $\bigotimes$ Antibodies \\
\hline & $\bigotimes$ Eukaryotic cell lines \\
\hline Х & Palaeontology and archaeology \\
\hline & $\bigotimes$ Animals and other organisms \\
\hline$\bigotimes$ & Human research participants \\
\hline$\bigotimes$ & Clinical data \\
\hline$\bigotimes$ & Dual use research of concern \\
\hline
\end{tabular}

Methods

\begin{tabular}{l|l}
\hline n/a & Involved in the study \\
$\square$ & $\bigotimes$ ChIP-seq \\
$\square$ & $\square$ Flow cytometry \\
$\square$ & $\square$ MRI-based neuroimaging
\end{tabular}


Antibodies used A detailed list of all antibodies used in the study is provided in the materials and methods. HP1 Developmental Studies Hybridoma Bank C1A9

HP1 Covance, PRB-291C-200

HA (C29F4) Cell Signaling \#3724S

HA.11 Covance MMS-101P

H3K9ac ActiveMotif 39586

H3K9ac ActiveMotif 39918

H3K9me3 ActiveMotif 39161

H3K9me2 ActiveMotif 39239

H3K9me3 Abcam ab176916

H3K27me3 Diagenode, C15410195 (Lot A1811-001P)

H3K4me1 Diagenode, C15410194 (Lot A1862D)

H3K4me3 Diagenode, C15410003

H3K27ac Diagenode, C15410196 (Lot A1723-041D)

Rpb3 (PollI) Carla Margulies Lab

Tubulin DM1 Sigma T9026

H3 ActiveMotif MABI 0301

Rpb3 (PollI) Asifa Akhtar Lab

Validation
Antibodies used in this study are commercially available and have been validated by the manufacturer. We further validated antibodies against H3K9me3, H3K9me2, H3K9ac, H3K27me3, H3K27ac, H3K4me1, HP1, Rpb3 either by Immunofluorescence staining or Western Blot in the control and the knockdown of the respective epigenetic writer or the protein itself.
HP1 Developmental Studies Hybridoma Bank C1A9 (validated by western blot and ChIP in HP1-KD in ED Fig. 1g and 3b)
HP1 Covance, PRB-291C-200
HA (C29F4) Cell Signaling \#3724S (according to manufacturer used in 866 oublications, validated for ChIP)
HA.11 Covance MMS-101P
H3K9ac ActiveMotif 39586 (according to manufacturer used in 7 publications, validated for ChIP)
H3K9ac ActiveMotif 39918 (according to manufacturer validated for ChIP and NGS applications)
H3K9me3 ActiveMotif 39161 (according to manufacturer validated for ChIP and NGS applications)
H3K9me2 ActiveMotif 39239 (validated by immunofluorescence in K9M in ED Fig. 4a)
H3K9me3 Abcam ab176916 (according to manufacturer used in 11 publications, validated for ChIP)
H3K27me3 Diagenode, C15410195 (Lot A1811-001P) (validated by ChIP in E(z)-KD in Zenk et al. 2017, Science)
H3K4me1 Diagenode, C15410194 (Lot A1862D) (according to manufacturer used in 44 publications, validated for ChIP)
H3K4me3 Diagenode, C15410003 (according to manufacturer used in 184 publications, validated for ChIP)
H3K27ac Diagenode, C15410196 (Lot A1723-041D) (according to manufacturer validated for ChIP and NGS applications)
Rpb3 (PollI) Carla Margulies Lab
Tubulin DM1 Sigma T9026
H3 ActiveMotif MABI 0301
Rpb3 (PollI) Asifa Akhtar Lab (validated by western blot in Rpb3 KD embryos)

\section{Eukaryotic cell lines}

Policy information about cell lines

Cell line source(s)

We used S2 cell line provided by Dr. Michael Boutros, DKFZ, Heidelberg. Details about the cell line are included in the materials and methods. Origin of S2 cells Schneider I (1972). "Cell Lines Derived from Late Embryonic Stages of Drosophila melanogaster". J. Embryol. Exp. Morphol. 27: 363-365.

Authentication

The cell line has been authenticated by visual inspection of the morphology. Further total RNA-Sequencing indicated absence of viral or microbial infections.

Mycoplasma contamination

The cell line tested negative for Mycoplasma.

Commonly misidentified lines

(See ICLAC register)

No commonly misidentified cell lines were used in the study.

\section{Palaeontology and Archaeology}

Specimen provenance

Provide provenance information for specimens and describe permits that were obtained for the work (including the name of the issuing authority, the date of issue, and any identifying information).

Specimen deposition

Indicate where the specimens have been deposited to permit free access by other researchers.

Dating methods

If new dates are provided, describe how they were obtained (e.g. collection, storage, sample pretreatment and measurement), where they were obtained (i.e. lab name), the calibration program and the protocol for quality assurance OR state that no new dates are provided.

Tick this box to confirm that the raw and calibrated dates are available in the paper or in Supplementary Information.

Ethics oversight

Identify the organization(s) that approved or provided guidance on the study protocol, OR state that no ethical approval or guidance 


\section{Animals and other organisms}

Policy information about studies involving animals; ARRIVE guidelines recommended for reporting animal research

Laboratory animals We used Drosophila melanogaster embryos to perform all experiments presented. We used fly lines encoding short hairpin RNAs against the target provided by the TRiP consortium and made available through the Bloomington stock center (Driver \#7063, HP1-KD \#33400). A description of fly lines generated in this study is provided in the materials and methods. All work was conducted in Drosophila melanogaster, an invertebrate animal (hexapod arthropod from the insect group). Invertebrate models are not regulated by the TierSchVersV and therefore are not subjected to ethical approval.

\section{Wild animals The study did not involve wild animals.}

Field-collected samples The study did not involve samples collected from the field.

Ethics oversight All work was conducted in Drosophila melanogaster, an invertebrate animal (hexapod arthropod from the insect group). Invertebrate models are not regulated by the TierSchVersV and therefore are not subjected to ethical approval.

Note that full information on the approval of the study protocol must also be provided in the manuscript.

\section{Human research participants}

Policy information about studies involving human research participants

Population characteristics

Describe the covariate-relevant population characteristics of the human research participants (e.g. age, gender, genotypic information, past and current diagnosis and treatment categories). If you filled out the behavioural \& social sciences study design questions and have nothing to add here, write "See above."

Recruitment

Describe how participants were recruited. Outline any potential self-selection bias or other biases that may be present and how these are likely to impact results.

Ethics oversight

Identify the organization(s) that approved the study protocol.

Note that full information on the approval of the study protocol must also be provided in the manuscript.

\section{Clinical data}

Policy information about clinical studies

All manuscripts should comply with the ICMJE guidelines for publication of clinical research and a completed CONSORT checklist must be included with all submissions.

Clinical trial registration

Study protocol

Data collection

Outcomes
Provide the trial registration number from ClinicalTrials. gov or an equivalent agency.

Note where the full trial protocol can be accessed OR if not available, explain why.

\section{Dual use research of concern}

Policy information about dual use research of concern

Hazards

Could the accidental, deliberate or reckless misuse of agents or technologies generated in the work, or the application of information presented in the manuscript, pose a threat to:

\footnotetext{
No $\mid$ Yes

$\square$ Public health

$\square \square$ National security

$\square \square$ Crops and/or livestock

$\square \square$ Ecosystems

$\square \square$ Any other significant area
} 


\section{Experiments of concern}

Does the work involve any of these experiments of concern:
No $\mid$ Yes
$\square$ Demonstrate how to render a vaccine ineffective
$\square$ Confer resistance to therapeutically useful antibiotics or antiviral agents
Enhance the virulence of a pathogen or render a nonpathogen virulent
$\square$ Increase transmissibility of a pathogen
$\square$ Alter the host range of a pathogen
Enable evasion of diagnostic/detection modalities
Enable the weaponization of a biological agent or toxin
$\square$ Any other potentially harmful combination of experiments and agents

ChIP-seq

\section{Data deposition}

$\bigotimes$ Confirm that both raw and final processed data have been deposited in a public database such as GEO.

$\bigotimes$ Confirm that you have deposited or provided access to graph files (e.g. BED files) for the called peaks.

Data access links

May remain private before publication

Files in database submission
All HiC, ChIP and RNA-Seq raw files generated in this study has been uploaded to GEO (GSE140542). https:// www.ncbi.nlm.nih.gov/geo/query/acc.cgi?acc=GSE140542

GSM4173897 ChIP H3K27ac IP repl1

GSM4173898 ChIP H3K27ac, H3K4me1 and H3K4me3 input repl1

GSM4173899 ChIP H3K27ac IP repl2

GSM4173900 ChIP H3K27ac and H3K4me3 input repl2

GSM4173901 ChIP H3K27me3 IP repl1

GSM4173902 ChIP H3K27me3 and Pol2 input repl1

GSM4173903 ChIP H3K27me3 IP repl2

GSM4173904 ChIP H3K27me3 and Pol2 input repl2

GSM4173905 ChIP H3K4me1 IP repl2

GSM4173906 ChIP H3K4me1 input repl2

GSM4173907 ChIP H3K4me1 IP repl1

GSM4173908 ChIP H3K4me3 IP repl1

GSM4173909 ChIP H3K4me3 IP repl2

GSM4173910 ChIP H3K9ac input repl1

GSM4173911 ChIP H3K9ac IP repl1

GSM4173912 ChIP H3K9ac IP repl2

GSM4173913 ChIP H3K9me3 IP repl1

GSM4173914 ChIP H3K9me3 input repl1

GSM4173915 ChIP H3K9me3 IP repl2

GSM4173916 ChIP H3K9me3 input repl2

GSM4173917 ChIP HP1 IP repl1

GSM4173918 ChIP HP1 input repl1

GSM4173919 ChIP HP1 input repl2

GSM4173920 ChIP HP1 IP repl2

GSM4173921 ChIP HP1 input repl3

GSM4173922 ChIP HP1 IP repl3

GSM4173923 ChIP HP1 IP repl1 Flag HA

GSM4173924 ChIP HP1 input repl1 Flag HA

GSM4173925 ChIP HP1 IP repl1 DSG

GSM4173926 ChIP HP1 input repl1 DSG

GSM4173927 ChIP Pol2 IP repl1

GSM4173928 ChIP Pol2 IP repl2

GSM4173929 HiC WT stage 5 repl1

GSM4173930 HiC WT stage 5 repl2

GSM4173931 HiC WT stage 5 repl3

GSM4173932 HiC WT stage 5 repl4

GSM4173933 HiC WT stage 5 repl5

GSM4173934 HiC WT stage 5 repl6

GSM4173935 HiC WT stage 5 repl7

GSM4173936 HiC HP1 KD stage 5 repl 1

GSM4173938 HiC HP1 KD stage 5 repl 3

GSM4173939 HiC HP1 KD stage 5 repl 4

GSM4173940 HiC HP1 KD stage 5 repl 5

GSM4173941 HiC HP1 KD + rescued stage 5 repl 1

GSM4173942 HiC HP1 KD + rescued stage 5 repl 2 
GSM4173943 HiC HP1 KD + rescued stage 5 repl 3

GSM4173944 HiC K9M stage 5 repl1

GSM4173946 RNA-seq WT repl1

GSM4173947 RNA-seq WT repl2

GSM4173948 RNA-seq WT repl3

GSM4173949 RNA-seq WT repl4

GSM4173950 RNA-seq HP1 KD repl1

GSM4173951 RNA-seq HP1 KD repl2

GSM4173952 RNA-seq HP1 KD repl3

GSM4576008 HiC HP1 KD stage 5 repl 2

GSM4576009 HiC K9M stage 5 repl2

GSM4594789 HiC WT S2 cells repl 1

GSM4594790 HiC WT S2 cells repl 2

GSM4594791 HiC HP1 KD rnai1 S2 cells repl 1

GSM4594792 HiC HP1 KD rnai1 S2 cells repl 2

GSM4594793 HiC HP1 KD rnai2 S2 cells repl 1

GSM4594794 HiC HP1 KD rnai2 S2 cells repl 2

GSM4595523 ChIP HP1 IP repl1 Covance

GSM4595524 ChIP HP1 Input repl1 Covance

GSM4595525 ChIP HP1 IP repl2 Covance

GSM4595526 ChIP HP1 Input repl2 Covance

GSM4595527 ChIP HP1 Input repl1 before cycle 9 with spike in

GSM4595528 ChIP HP1 IP repl1 before cycle 9 with spike in

GSM4595529 ChIP HP1 Input repl2 before cycle 9 with spike in

GSM4595530 ChIP HP1 IP repl2 before cycle 9 with spike in

GSM4595531 ChIP HP1 Input repl1 cycle 9-13 with spike in

GSM4595532 ChIP HP1 IP repl1 cycle 9-13 with spike in

GSM4595533 ChIP HP1 Input repl2 cycle 9-13 with spike in

GSM4595534 ChIP HP1 IP repl2 cycle 9-13 with spike in

GSM4595535 ChIP HP1 Input repl1 stg5 HP1KD embryo with spike in

GSM4595536 ChIP HP1 IP repl1 stg5 HP1KD embryo with spike in

GSM4595537 ChIP HP1 Input repl2 stg5 HP1KD embryo with spike in

GSM4595538 ChIP HP1 IP repl2 stg5 HP1KD embryo with spike in

GSM4595539 ChIP HP1 Input repl1 stg5 K9M embryo with spike in

GSM4595540 ChIP HP1 IP repl1 stg5 K9M embryo with spike in

GSM4595541 ChIP HP1 Input repl2 stg5 K9M embryo with spike in

GSM4595542 ChIP HP1 IP repl2 stg5 K9M embryo with spike in

GSM4595543 ChIP HP1 Input repl1 stg5 WT embryo with spike in

GSM4595544 ChIP HP1 IP repl1 stg5 WT embryo with spike in

GSM4595545 ChIP HP1 Input repl2 stg5 WT embryo with spike in

GSM4595546 ChIP HP1 IP repl2 stg5 WT embryo with spike in

GSM4983387 ChIP HP1 IP repl1 in ovaries

GSM4983388 ChIP HP1 Input repl1 in ovaries

GSM4983389 ChIP HP1-CD IP repl1 in ovaries

GSM4983390 ChIP HP1-CD Input repl1 in ovaries

GSM4983391 ChIP HP1 IP repl2 in ovaries

GSM4983392 ChIP HP1 Input repl2 in ovaries

GSM4983393 ChIP HP1-CD IP repl2 in ovaries

GSM4983394 ChIP HP1-CD Input repl2 in ovaries

Genome browser session

(e.g. $\underline{\operatorname{UCSC}}$ )

All bigwig files are available in GEO (GSE140542).

\section{Methodology}

Replicates

All ChIP experiments were performed in biological replicates. We inspected the agreement of replicates visually in the genome browser and by computing heatmaps on the individual replicates.

Sequencing depth

We sequenced all samples in the study at least to a depth of 15 Mio.

Antibodies

A detailed list of all antibodies used in the study is provided in the materials and methods (table 1). The HP1 antibody was also validated by performing ChIP-seq in HP1-KD embryos.

Peak calling parameters Peaks were called using macs2, using a cut-off of 0.05 on the q-value. --broad option was added for the H3K9me3 and HP1 ChIP. Further details are available in the materials and methods.

Data quality

We visually inspected all ChIP tracks and called peaks in the genome browser. To call the peaks we used a cut-off of 0.05 on the $\mathrm{q}$ value and only included peaks that passed this threshold.

Software

Reads were mapped to the D. melanogaster genome (build dm6) using qAlign from QuasR package using the following alignments parameters: -n 2 -l 28 -e $70-\mathrm{k} 1$-X 500. This allows to keep reads mapping to multiple loci and randomly assigns them to one of the multiple locations. ChIP enrichment over input was calculated using bamCompare from deepTools using the following parameters: -scaleFactorsMethod SES --smoothLength 900 --binSize 300. Further details are available in the materials and methods. 


\section{Plots}

Confirm that:

$\square$ The axis labels state the marker and fluorochrome used (e.g. CD4-FITC).

$\square$ The axis scales are clearly visible. Include numbers along axes only for bottom left plot of group (a 'group' is an analysis of identical markers).

$\square$ All plots are contour plots with outliers or pseudocolor plots.

A numerical value for number of cells or percentage (with statistics) is provided.

\section{Methodology}

Sample preparation

Describe the sample preparation, detailing the biological source of the cells and any tissue processing steps used.

Instrument

Identify the instrument used for data collection, specifying make and model number.

Software

Describe the software used to collect and analyze the flow cytometry data. For custom code that has been deposited into a community repository, provide accession details.

Cell population abundance

Describe the abundance of the relevant cell populations within post-sort fractions, providing details on the purity of the samples and how it was determined.

Gating strategy

Describe the gating strategy used for all relevant experiments, specifying the preliminary FSC/SSC gates of the starting cell

population, indicating where boundaries between "positive" and "negative" staining cell populations are defined.

$\square$ Tick this box to confirm that a figure exemplifying the gating strategy is provided in the Supplementary Information.

\section{Magnetic resonance imaging}

\section{Experimental design}

Design type

Design specifications

Behavioral performance measures

Indicate task or resting state; event-related or block design.

Specify the number of blocks, trials or experimental units per session and/or subject, and specify the length of each tria or block (if trials are blocked) and interval between trials.

State number and/or type of variables recorded (e.g. correct button press, response time) and what statistics were used to establish that the subjects were performing the task as expected (e.g. mean, range, and/or standard deviation across subjects).

\section{Acquisition}

Imaging type(s)

Specify: functional, structural, diffusion, perfusion.

Field strength

Sequence \& imaging parameters

Area of acquisition

Specify in Tesla

Specify the pulse sequence type (gradient echo, spin echo, etc.), imaging type (EPI, spiral, etc.), field of view, matrix size, slice thickness, orientation and TE/TR/flip angle.

State whether a whole brain scan was used OR define the area of acquisition, describing how the region was determined.

Diffusion MRI

Used

Not used

\section{Preprocessing}

Preprocessing software

Normalization

Normalization template

Noise and artifact removal

Provide detail on software version and revision number and on specific parameters (model/functions, brain extraction, segmentation, smoothing kernel size, etc.).

If data were normalized/standardized, describe the approach(es): specify linear or non-linear and define image types used for transformation OR indicate that data were not normalized and explain rationale for lack of normalization.

Describe the template used for normalization/transformation, specifying subject space or group standardized space (e.g. original Talairach, MNI305, ICBM152) OR indicate that the data were not normalized.

Describe your procedure(s) for artifact and structured noise removal, specifying motion parameters, tissue signals and physiological signals (heart rate, respiration). 


\section{Statistical modeling \& inference}

Model type and settings

Specify type (mass univariate, multivariate, RSA, predictive, etc.) and describe essential details of the model at the first and second levels (e.g. fixed, random or mixed effects; drift or auto-correlation).

Effect(s) tested

Define precise effect in terms of the task or stimulus conditions instead of psychological concepts and indicate whether ANOVA or factorial designs were used.

Specify type of analysis:

ROI-based

Both

Statistic type for inference

(See Eklund et al. 2016)

Correction

Specify voxel-wise or cluster-wise and report all relevant parameters for cluster-wise methods.

Describe the type of correction and how it is obtained for multiple comparisons (e.g. FWE, FDR, permutation or Monte Carlo)

Models \& analysis

$\mathrm{n} / \mathrm{a}$ Involved in the study

$\square \square$ Functional and/or effective connectivity

$\square$ Graph analysis

$\square$ Multivariate modeling or predictive analysis

Functional and/or effective connectivity

Report the measures of dependence used and the model details (e.g. Pearson correlation, partial correlation, mutual information).

Graph analysis

Report the dependent variable and connectivity measure, specifying weighted graph or binarized graph, subject-or group-level, and the global and/or node summaries used (e.g. clustering coefficient, efficiency, etc.)

Multivariate modeling and predictive analysis

Specify independent variables, features extraction and dimension reduction, model, training and evaluation metrics. 